\title{
TITLE
}

\section{The Androgen Receptor: A Therapeutic Target in Desmoplastic Small Round Cell Sarcoma}

Salah-Eddine Lamhamedi-Cherradi ${ }^{1, *, \#}$, Mayinuer Maitituoheti ${ }^{2, *}$, Brian A. Menegaz ${ }^{3}$, Sandhya Krishnan ${ }^{1}$, Amelia M. Vetter ${ }^{1}$, Pamela Camacho ${ }^{4}$, Chia-Chin Wu ${ }^{2}$, Hannah C. Beird ${ }^{2}$, Robert W. Porter ${ }^{1}$, Davis R. Ingram ${ }^{5}$, Vandhana Ramamoorthy ${ }^{2}$, Sana Mohiuddin ${ }^{6}$, David McCall ${ }^{6}$, Danh D. Truong ${ }^{1}$, Branko Cuglievan ${ }^{6}$, P. Andrew Futreal $^{5}$, Alejandra Ruiz Velasco ${ }^{1}$, Nazanin Esmaeili Anvar ${ }^{5}$, Budi Utama ${ }^{7}$, Mark Titus ${ }^{8}$, Alexander J. Lazar ${ }^{5}$, WeiLien Wang ${ }^{5}$, Cristian Rodriguez-Aguayo ${ }^{9}$, Ravin Ratan ${ }^{1}$, John A. Livingston ${ }^{1}$, Kunal Rai ${ }^{2, \#}$, A. Robert MacLeod ${ }^{10}$, Najat C. Daw ${ }^{6}$, Andrea Hayes-Jordan ${ }^{11}$, and Joseph A. Ludwig ${ }^{1, \# .}$

\section{ONE SENTENCE SUMMARY}

We demonstrate that DSRCT, an aggressive pediatric cancer, is an AR-driven malignancy capable of responding to androgen deprivation therapy.

\section{AUTHOR AFFILIATIONS}

${ }^{1}$ Sarcoma Medical Oncology department, The University of Texas MD Anderson Cancer Center, Houston, Texas 77005; '2Department of Genomic Medicine, The University of Texas MD Anderson Cancer Center, Houston, Texas 77005; ${ }^{3}$ Department of Surgery, Breast surgical Oncology, Baylor College of Medicine, Houston, Texas, 77030; ${ }^{4}$ Texas Children's Cancer \& hematology Centers, Houston, Texas 77384; ${ }^{5}$ Division of Pathology, ${ }^{6}$ Division of Pediatrics, The University of Texas MD Anderson Cancer Center, Houston, Texas 77005; ${ }^{7}$ Optical Microscopy Facility, Rice University, Houston 77030 TX ${ }^{8}$ Genitourinary Medical Oncology Department, The University of Texas MD Anderson Cancer Center, Houston, Texas 77005; ${ }^{9}$ Experimental Therapeutics Department, The University of Texas MD Anderson Cancer Center, Houston, Texas 77005; ${ }^{10}$ Ionis Pharmaceuticals, Carlsbad, CA 92010; and ${ }^{11}$ Lineberger Comprehensive Cancer Center, UNC, Chapel Hill, NC 27599.

${ }^{*}$ Co-first authors

Running Title: Targeting the androgen receptor in DSRCT

Word Count: Abstract (250); Manuscript body (9449)

Key Words: DSRCT, sarcoma, pediatric cancer, fusion protein, EWS-WT1, androgen receptor (AR)

"Corresponding Authors:

Salah-Eddine Lamhamedi-Cherradi, Ph.D.

Project Director

Department of Sarcoma Medical Oncology

University of Texas MD Anderson Cancer Center

1515 Holcombe Blvd, Houston, Texas, 77005

Tel: +1 (713) 792-3626

Email: SLamhamedi@mdanderson.org

Kunal Rai, Ph.D.

Associate Professor

Department of Genomic Medicine

University of Texas MD Anderson Cancer Center

1515 Holcombe Blvd, Houston, Texas, 77005

Tel: +1 (713) 792-6809

Email: Krai@mdanderson.org
Joseph A. Ludwig, MD

Associate Professor

Department of Sarcoma Medical Oncology

University of Texas MD Anderson Cancer Center

1515 Holcombe Blvd, Houston, Texas, 77005

Tel: +1 (713) 792-3626

jaludwig@mdanderson.org

Financial Disclosures: Dr. MacLeod is an employee and shareholder of lonis Pharmaceuticals.

Research Support: The University of Texas MD Anderson Cancer Center is supported by the National Institutes of Health through Cancer Center Support Grant CA016672. JAL and AHJ are funded through the generous philanthropy of the Cory Monzingo Foundation. 


\section{ABSTRACT}

Desmoplastic small round cell tumor (DSRCT) is an aggressive, usually incurable sarcoma subtype that predominantly occurs in post-pubertal young males. Recent evidence suggests that the androgen receptor (AR) can promote tumor progression in DSRCTs. However, the mechanism of AR-induced oncogenic stimulation remains undetermined. Herein, we demonstrate that enzalutamide and AR-directed antisense oligonucleotides (AR-ASO) block $5 \alpha$-dihydrotestosterone (DHT)-induced DSRCT cell proliferation and reduce xenograft tumor burden. Gene expression analysis and chromatin immunoprecipitation sequencing (ChIP-seq) were performed to elucidate how AR signaling regulates cellular epigenetic programs. Remarkably, ChIP-seq revealed novel DSRCT-specific AR DNA binding sites adjacent to key oncogenic regulators, including WT1 (the C-terminal partner of the pathognomonic fusion protein) and FOXF1. Additionally, AR occupied enhancer sites that regulate the Wnt pathway, neural differentiation, and embryonic organ development, implicating AR in dysfunctional cell lineage commitment. Our findings have immediate clinical implications given the widespread availability of FDAapproved androgen-targeted agents used for prostate cancer. 
bioRxiv preprint doi: https://doi.org/10.1101/2022.01.20.477146; this version posted January $22,2022$. The copyright holder for this preprint

(which was not certified by peer review) is the author/funder, who has granted bioRxiv a license to display the preprint in perpetuity. It is made available under aCC-BY-NC-ND 4.0 International license.

\section{INTRODUCTION}

Desmoplastic small round cell tumor (DSRCT) is an aggressive soft-tissue malignancy that usually presents in post-pubertal adolescents and young adults as a large intra-abdominal mass together with widespread coating of serosal and subdiaphragmatic surfaces by hundreds to thousands of malignant nodules. Given the inconspicuous tumor location, nearly all patients present in an advanced-stage with symptoms of abdominal pain or distention, nausea, constipation, and weight loss'1.

DSRCT's cell of origin is unknown, and these tumors universally exhibit a high-grade, poorly differentiated state characterized by cellular nests of unclear lineage, seeming to show epithelial, muscular, mesenchymal, and neural differentiation admixed with prominent desmoplastic stroma ${ }^{2-4}$. Given its rarity, with an age-adjusted incidence peak incidence of 0.3-0.74 cases per million, ${ }^{5}$ it wasn't until 1989 that Gerald and Rosai first described DSRCT as a unique clinicopathologic disease ${ }^{6}$. Shortly thereafter, cytogenetic analyses demonstrated that DSRCT tumors harbor a pathognomonic $\mathrm{t}(11 ; 22)(\mathrm{p} 13: \mathrm{q} 12)$ chromosomal translocation that pairs the Ewing sarcoma (ES) gene (EWSR1) with the Wilms tumor suppressor gene $(W T 1)^{6-9}$. The resulting chimeric $59 \mathrm{kDa}$ fusion protein (FP), in concert with the heterozygous functional loss of the WT1 tumor suppressor protein, promotes an oncogenic effect that reinforces tumor survival and growth ${ }^{10}$.

The PI3K/AKT and androgen receptor (AR) signaling cascades are among the most frequently activated in cancer11,12. Given the striking observation that $90 \%$ of DSRCT cases occur in post-pubertal males (with an average age at diagnosis of 21.4 years), we investigated how AR contributes to tumorigenesis and survival4,13,14. A potential connection between the AR and DSRCT, first reported by Fine et al. in 2006, studied a series of twenty-seven advanced-stage DSRCT patients who had progressed through at least two chemotherapy regimens ${ }^{15}$. In that retrospective multi-center analysis, $37 \%$ of the samples were $\geq 2+$ by immunohistochemistry, and surprisingly, three of six $\mathrm{AR}^{+}$patients transiently benefited from $1^{\text {st-generation combined androgen blockade }}$ (CAB) using Lupron and bicalutamide. Despite that promising signal of activity, AR-targeting has not been pursued further in the clinic, and at the time of this writing, no additional studies were reported in the literature.

In the present work, we use DSRCT xenografts and patient-derived tumor explants (PDXs) to extend the findings by Fine et al. to modern-day AR-targeted therapies, such as enzalutamide, that form the backbone of prostate cancer treatment ${ }^{16}$. Additionally, we present promising efficacy data using an experimental AR-targeted antisense oligonucleotide (ASO) that significantly delayed tumor growth by suppressing AR expression. Finally, we present chromatin immunoprecipitation sequencing (ChIP-seq) results that suggest a new mechanistic understanding of AR's role in DSRCT tumorgenicity. Our research findings substantiate DSRCT as a second AR-driven malignancy, have direct implications for DSRCT treatment, and implicitly suggest a path towards clinical trials that center on AR-directed treatment options for this otherwise intractable pediatric cancer.

\section{RESULTS}

\section{Protein expression in DSRCT differs substantially from ES}

Given the differences in clinical presentation, tumor biology, and response to biologically targeted therapies, we conducted a reverse-phase protein lysate array (RPPA) to identify proteins enriched in DSRCT. To determine tumor-specific proteins, we compared protein lysates from DSRCT nodules and paired adjacent normalappearing mesenteric tissue from the same patients using a well-described RPPA platform enriched for known oncoproteins (Fig. S1) ${ }^{17-19}$. Unsupervised double hierarchical clustering correctly separated normal mesenteric tissues from DSRCT, which over-expressed Akt, Syk, PKC- $\alpha$, and other proteins. Next, we identified proteins enriched in DSRCT compared to another malignancy, using ES as the closest molecular cousin that shares an $\mathrm{N}$-terminus EWSR1 fusion partner. Interestingly, AR and Syk proteins were upregulated in most DSRCT specimens but nearly undetectable in ES (Fig 1A-B). The RPPA data, validated by Western blots, provides the first screen of proteins enriched in DSRCT (Fig. 1C-D). 


\section{Expression of AR in DSRCT primary tumors}

Since AR activity requires androgen-mediated nuclear translocation, we created a DSRCT-specific tissue microarray (TMA) from sixty cases treated at MD Anderson Cancer Center (MDACC) to determine the prevalence and cellular distribution of AR staining in patients treated at a single institution. Seventy-five percent of the cores available for analysis were positive for nuclear AR by immunohistochemistry (Fig. 2A-B and Fig. $\mathrm{S} 2 \mathrm{~A}-\mathrm{C}$ ), usually more prominently in the epithelioid cells rather than the desmoplastic stromal cells. Of the ARpositive samples, $7 \%$ showed focal AR expression in $10-50 \%$ rare, scattered cells and could be of any intensity (low positive AR expression), 3.3\% had low AR expression where only $1-10 \%$ of cells are positive for AR expression (focal expression), and $25 \%$ demonstrated negative AR expression (where only $0-1 \%$ of cells are positive). $65 \%$ showed high AR expression, defined by AR-positivity in $>50 \%$ of a sample's cells (Fig. $2 \mathrm{~B}$ and Fig. S2C). High-intensity staining was defined as completely obscuring the nuclear hematoxylin counterstain, while moderate staining allowed visualization of the stain. Weak staining required examination of the cells at least 200X to detect staining reliably. As an additional metric of AR expression, we evaluated an additional 12 DSRCT patient tumors for AR expression by Western blotting: $42 \%$ of the tumors showed high AR expression, $33 \%$ had moderate AR expression, and $25 \%$ were AR-negative (Fig. 2C \& 2D). As IHC and Western blotting revealed moderate to high AR expression in three-quarters of the DSRCT cases assessed, this seemed to substantiate the RPPA results.

\section{Genomic profiling of DSRCT, PC, and other sarcoma primary tumor samples}

Given the male predominance of DSRCT, new AR protein expression data, and known abundance of ARtargeted therapies used for prostate cancer, we elected to pursue AR as a potential therapeutic target in DSRCT. The case report by Fine et al. hinted that DSRCT patients can respond briefly to a $1^{\text {st }}$-generation CAB, which heightened our enthusiasm to investigate how AR signaling contributes to DSRCT biology ${ }^{15}$.

Given the AR's central role in prostate cancer (PC) growth and survival, and deep mechanistic understanding in that malignancy ${ }^{20-23}$, we performed a gene expression analysis comparing 22 DSRCT samples to 12 PC samples and a group of other diverse sarcoma subtypes, including 7 chondrosarcomas, 7 well-differentiated liposarcomas (WDLPS), 10 dedifferentiated liposarcoma (DDLPS), and 47 osteosarcoma samples, which served as negative controls. As expected, DSRCT demonstrated significant AR upregulation compared to these other sarcoma samples from chondrosarcoma, well-differentiated and dedifferentiated liposarcoma, and osteosarcoma $(p<0.01-p<0.0001)$ but did not surpass the levels observed in PC (Fig. 2E-F).

Compared to other sarcoma subtypes, most DSRCT samples clustered together based upon their expression of 89 genes associated with the androgen pathway, as defined by KEGG (Fig. S2D). Similarly, DSRCT samples clustered together by their expression of 55 genes linked to canonical androgen signaling (Supplemental Fig. $2 \mathrm{~F})$. Pathway analysis revealed other cancer pathways enriched in DSRCT compared to other sarcoma subtypes. This included the cell adhesion molecule (CAM) communication pathway that regulates cell invasion through a coordinated balance between adhesion and detachment of cells $\mathrm{s}^{24,25}$ and the extracellular matrix (ECM) interaction pathway, known to initiate cell motility across the ECM barrier ${ }^{26}$.

\section{Characterizing extranuclear partners of $A R$ and its nuclear cofactors}

AR splice variants (AR-Vs) have been implicated in PC tumor progression, an increased risk of biochemical relapse, and inferior overall survival outcomes ${ }^{27-29}$. To determine if variant forms of AR exist in DSRCT, we assessed AR-V7 expression within a cohort of twelve consecutive DSRCT patients. As none of the initial twelve samples expressed AR-V by IHC, we elected not to examine this further in the broader sample set.

As AR activity can be influenced by integrins and transcriptional co-regulators ${ }^{30,31}$, we evaluated these AR activators within the same cohort of 11 DSRCT tumors at the proteomic level using a western blotting analysis. Among three alpha integrin (ITGAV, ITGA4, and ITGA5) and beta integrin (ITGB1, ITGB3, and ITGB5) subunits commonly observed in mesenchymal tissues, protein expression varied considerably and did not correlate with AR expression (Fig. S3A). 
bioRxiv preprint doi: https://doi.org/10.1101/2022.01.20.477146; this version posted January $22,2022$. The copyright holder for this preprint

(which was not certified by peer review) is the author/funder, who has granted bioRxiv a license to display the preprint in perpetuity. It is made available under aCC-BY-NC-ND 4.0 International license.

Since the epigenetic effects of AR can be modified by cofactor binding and matrix metalloproteins, we assessed whether steroid receptor coactivators NCOA1/2/3 or MMP2/13 contribute to the development of DSRCT through AR-dependent mechanisms ${ }^{32-37}$. To accomplish this, we performed a Western blot of 11 primary DSRCT tumors and the JN-DSRCT and LNCaP PC cell lines. The three-NCOA biomarkers demonstrated heterogeneous expression in the DSRCT clinical samples proportional to their AR expression (Fig. S3B). However, the JN-DSRCT cells showed low expression of NCOA1/2 and equivalent expression of NCOA2 versus LNCaP PC cells (Fig. S3C). Further investigation with a larger sample set will be required to determine how the AR-dependent integrin/NCOA-dependent pathway impacts DSRCT cell migration and death.

\section{In vitro stimulation and inhibition of DSRCT proliferation via AR}

Though AR activation by testosterone and DHT leads to brisk PC cell proliferation ${ }^{38}$, it was uncertain whether DSRCT cells similarly relied upon AR signaling for proliferation, growth, and survival. To evaluate this, we performed in vitro cell proliferation assays following DHT-mediated AR stimulation in JN-DSRCT; AR-expressing LNCaP PC cells, AR-non-expressing PC3 PC cells, and ES TC71 cells that were used as positive or negative controls. As hypothesized, DHT stimulation increased cell proliferation of JN-DSRCT and LNCaP cells compared to PC3 and ES cells (Fig. 3A). As measured by Western blotting, we confirmed strong AR expression by LNCaP and JN-DSRCT cell lines following DHT stimulation in contrast to its absence in the TC71 ES and PC3 prostate cancer cells (Fig. 3B). Next, we performed confocal immunofluorescence staining of these cells to determine if (and how quickly) DHT-mediated stimulation would facilitate AR transmigration from the cytoplasm into the cell nucleus. Our results suggest that AR-upregulation begins within 5 hours of DHT exposure and peaks in JNDSRCT, or decreases in LNCaP cells at 24 hours (Fig. 3C-D).

Having shown that DHT stimulates DSRCT cells, we explored whether FDA-approved and experimental AR antagonists had an antiproliferative effect. Both enzalutamide (Fig. 3E) and the novel AR-ASO (IONIS 560131; formerly AZD5312) significantly slowed DSRCT cell proliferation at two weeks (Fig. 3F) and reduced AR expression (Fig. 3G). However, the in vitro antiproliferative effect was 4-fold more effective in the cells treated with the AR-targeted antisense blockade (Fig. 3E \& 3F). Notably, this antineoplastic effect required 72-hours of DHT pretreatment (Fig. S4). Altogether, this data indicates a vital role for DHT-stimulated AR expression in DSRCT cell proliferation and conclusively demonstrates a potent antineoplastic effect of AR antagonists.

\section{Preclinical efficacy of AR-based targeted therapy for the treatment of DSRCT.}

Since only one DSRCT cell line exists, we extended our evaluation of the AR antagonists to the in vivo setting using the JN-DSRCT xenograft and available DSRCT patient-derived tumor explants (PDXs). Immunocompromised NSG mice bearing JN-DSRCT xenograft tumors treated with enzalutamide or AR-ASO significantly reduced tumor burden and improved survival with the same efficacy, compared to placebo or control groups during the first two months of treatment (Fig. 4A-B). At two months, tumor growth began to accelerate in the enzalutamide-treated mice, whereas growth suppression continued in the mice treated with either 25 or 50 $\mathrm{mg} / \mathrm{kg}$ of the AR-ASO ( $\mathrm{p}<0.0001$; Fig. 4A). Compared to enzalutamide, the AR-targeted ASO (25 and $50 \mathrm{mg} / \mathrm{kg}$ ) demonstrated superior antineoplastic activity (Fig. 4A; $p<0.0001$ or $p=0.006$, respectively). The effects of ARASO and control ASOs were also assessed in NSG mice (5 mice/group) bearing a DSRCT PDX (Fig. 4D-F). As expected, tumor growth and Kaplan-Meier curves revealed that tumors treated with AR-ASO have significantly reduced tumor burden and improved survival compared to control ASO group ( $p=0.0097 \& p<0.0001$, respectively).

Though both agents delayed tumor growth, AR-ASOs were more effective than enzalutamide in both preclinical models. Therefore, our pharmacodynamic analysis focused primarily on the effect of AR-ASO treatment. Proteomic profiling by RPPA (Fig. 5A), Western blotting (Fig. 5B \& C), immunofluorescence (Fig. 5D \& E \& Fig. S5A-C), and immunohistochemistry (Fig. 5F-H \& Fig. S5D-F) validated the AR-ASO mediated knockdown of AR expression in the xenograft and PDX. To further characterize how the AR-ASO differed from enzalutamide mechanistically, we performed liquid chromatography tandem mass spectrometry (LC/MS/MS) analysis of 38 collected preclinical animal specimens shown in Figure 4. Consistent with prior literature in PC, 
loss of AR following AR-ASO treatment destabilized testosterone and reduced its intratumoral expression (Fig. $\mathrm{S} 6)^{39,40}$. As a negative control, the corticosterone levels were unchanged by AR-blockade. Additionally, since the antineoplastic action of enzalutamide works by preventing ligand-AR binding, reducing AR shuttling to the nucleus, and impairing AR DNA binding affinity - instead of reducing AR levels (Fig. S7; panels A-E) enzalutamide-treatment did not significantly lower intratumoral testosterone.

To gain a preliminary understanding of the short-term pharmacodynamic effects of AR suppression, a group of JN-DSRCT xenografts and DSRCT PDXs was collected 10 days into their AR-ASO treatment (Fig. 5A ARASO PD) for analysis by RPPA to assess early compensatory pharmacodynamic changes. pS6, Akt, ER, PD$1 \mathrm{~L}, \mathrm{pAKT}$, and other proteins (Figure 5A and Supplemental Figure 7F-G) were upregulated. Others have reported that the PI3K-AKT pathway has pleiotropic effects in survival, proliferation, metabolism, and growth pathways of several malignancies ${ }^{41}$, and its blockade has long been of interest in managing PC, where a compensatory increase in AKT signaling can occur following AR inhibition ${ }^{42}$. Notably, the same AR-ASO (AZD5312) used in our preclinical experiments was well-tolerated when administered to PC patients (NCT03300505). Therefore, one could theoretically investigate this AR-ASO drug candidate in DSRCT-specific phase 2 trials without delay. Given the limited nature of our preclinical studies, future studies with enzalutamide is also of interest.

\section{AR directly regulates important oncogenic regulators in DSRCT}

To model the AR transcriptional program in a human JN-DSRCT cell line, we determined the genome-wide AR binding profiles using ChIP-Seq experiments in unstimulated or DHT-stimulated JN-DSRCT cells treated with control ASO or AR-ASO. As expected, DHT treatment enhanced AR binding to the chromatin as assessed by the average intensity plot on all significant peaks $\left(p<1 e^{-7}\right)$ and heat map (Fig. 6A). DHT stimulation led to $\sim 4000$ new peaks that were suppressed by treatment with AR ASO (Fig. 6B and Supplemental Table 1). These binding sites were enriched at known AR response elements (AREs) and in sites for FOXA1, a transcription factor known to open compacted DNA and cooperate with AR in prostate cancer $^{43}$ (Fig. 6C and Supplemental Table 1). Consistent with DSRCT's pathogenesis, we also noted enrichment of WT1 binding motifs within AR binding peaks (Fig. 6C) suggesting potential interactions between AR, FOXA1, and WT1 in JN-DSRCT cells. To further characterize the genes adjacent to AR binding site peaks, we performed a pathway analysis using 700 genes that are direct targets of AR. Upregulated pathways included the TNF $\alpha$ pathway, Hippo signaling, and pluripotency regulators (Fig. 6D and Supplemental Table 1), and key genes included WT1, CTNNB1, SOX2, GLI2, FOXF1 and GATA6 (Fig. 6E, Fig. S8D and Supplemental Table 1).

After evaluating the effects of androgen stimulation and withdrawal in JN-DSRCT cells, we next compared DSRCT to data from PC cells. Significant overlap existed at sites for AR binding at AREs (Fig. S8A), FOXA1 motifs (Fig. S8B), and sites that regulate key cancer pathways, including WNT, TGF $\beta$, PI3K, MAPK, Hippo signaling, TNF $\alpha$ and epithelial-to-mesenchymal transformation (EMT) (Fig. S8C). To further evaluate the AR regulatory function in DSRCT tumor mouse models, we performed ChIP-seq on DSRCT-xenograft and PDX samples. Consistent with the cell line data, we observed suppressed AR binding to the chromatin by the treatment with AR ASO (Fig. S9A-B). Similarly, pathway analysis of the top 5000 lost AR binding sites targeted genes showed enrichment of MAPK pathway, Hippo signaling, Wnt signaling and pluripotency regulators (Fig. S9C-D). We also noted enrichment of AR and FOX family binding motifs within AR binding peaks in both DSRCTxenograft and PDX samples (Fig. S9E). Genes adjacent to AR binding site peaks also showed high overlap with DSRCT specific genes in both models (Fig. S9F). Key genes from cell line data (Fig.6E) also showed AR signal reduction after AR-ASO treatment (Fig. S9G).

\section{AR-dependent enhancer reprogramming activates oncogenic pathways in DSRCT}

Several studies have shown that AR establishes a pro-tumorigenic transcriptome by reprogramming the active enhancer landscape (assessed by H3K27ac profiles) in prostate cancer progression ${ }^{44}$. Therefore, we asked if AR plays similar roles in DSRCT by examining genome-wide profiles for H3K27ac marks in unstimulated or DHT stimulated JN-DSRCT cells treated with control ASO or AR-ASO. We noted that unstimulated cells treated with AR-ASO showed a higher intensity and a higher number of H3K27ac peaks compared to control ASO treated 
cells (Fig. 7A-B, S10A, and Supplemental Table 2). Similarly, AR-ASO treatment in DHT-treated cells also increased the active enhancer peaks compared to control ASO treatment (Fig. 7A-B, S10A, and Supplemental Table 2). This observation is contrary to those in prostate cancers where active enhancer peaks are positively associated with higher AR activity ${ }^{44}$. It has been previously shown that AR recruits the MLL complex and $\mathrm{CBP} / \mathrm{p} 300$, which is responsible for active enhancer marking in prostate cancer ${ }^{45}$. To identify which enhancers were likely derived by AR binding and potential recruitment of enhancer-marking proteins, we overlapped the AR and H3K27ac peaks in DHT treated cells (Fig. S10B and Supplemental Table 2). We then intersected these AR-targeted enhancer peaks with highly expressed genes in DSRCT (Fig. 7C and Supplemental Table 2) (FC> 1.5 , adjusted $p$-value $<0.05$ in comparison to other sarcoma subtypes). There, we identified WNT signaling and cell-adhesion as major drivers that are regulated at the chromatin level by AR-dependent active enhancer programs (Fig. 7D). The genes with direct AR binding and enhancer gains included important oncogenes such as AXIN2 and CDK6 (Fig. 7E). Additionally, we investigated alterations in super-enhancer (SE) regions that harbor a high-density of TF binding motifs ${ }^{46-48}$. SEs in control ASO treated cells marked important oncogenes such as AKT3 and GRHL2, whereas SEs in AR-ASO treated cells marked tumor suppressor genes such as RUNX1 and CUX1 (Fig. S10C-D and Supplemental Table 3), that potentially regulate the AR-driven transcriptome. Overall, our results suggest that AR activation reprograms typical enhancers and SE to regulate key oncogenic signaling pathways in DSRCT.

Interestingly, in preclinical tumor samples we also observed similar enhancer reprogramming. AR-ASO treatment of the DSRCT-xenograft significantly increased the active enhancer and promoter binding sites compared to control ASO treatment, whereas PDX samples showed a moderate increase (Fig. S11A-D). We also observed AR-dependent active enhancers regulating PI3K-AKT-mTOR, WNT signaling, cell-adhesion pathways (Fig. S11E-F), and key oncogenes (Fig. 7E), with direct AR binding and enhancer gains in both DSRCT-xenograft and PDX models (Fig. S11G).

\section{DISCUSSION}

Ever since Ladanyi and Gerald discovered the EWSR1-WT1 chromosomal translocation ${ }^{8}$, DSRCT has been treated with the same chemotherapy regimens used for ES. The recent exceptions include ES-specific agents like TK-216 that target c-terminus ETS genes (e.g., FLI1 or ERG), or pazopanib, which demonstrates preferential activity in DSRCT and other soft-tissue sarcomas ${ }^{49}$. Phase II studies testing neoantigen targeted monoclonal antibodies (Abs), for example 8H9 in DSRCT, are also directed at unique sarcoma subtypes ${ }^{50}$.

As three-quarters of all DSRCT patients typically succumb to their malignancy within 5-years, our RPPA study intended to define new molecular targets for DSRCT and expand our therapeutic arsenal of biologically targeted therapies that engage them (Figure 1). Surprisingly, of 151 proteins assessed in the RPPA, SYK and AR were the most differentially expressed. The SYK protein - not previously reported in DSRCT - is a nonreceptor tyrosine kinase (also known as spleen tyrosine kinase) commonly found in hematological tissues. Its constitutive activation has been shown to induce malignant transformation of B-cells to lymphomas or leukemias. As such, the oral SYK inhibitors cerdulatinib (Portola Pharmaceutical) and entospletinib (Gilead Sciences) are under active clinical investigation for the treatment of certain lymphomas, chronic lymphocytic leukemia, and acute myeloid leukemia (NCT01994382 and NCT02457598). An orally active SYK inhibitor, fostamatinib, has already received FDA-approval as a treatment for immune thrombocytopenia and continues to be investigated as an experimental therapy for hematological malignancies (NCT00446095). Though tantalizing to consider that SYK hyperactivation plays an oncogenic role in DSRCT, we have not yet had the opportunity to evaluate these relatively new drugs within our preclinical DSRCT models.

In contrast to SYK, numerous FDA-approved and experimental AR antagonists were available for immediate preclinical evaluation, and potentially available to patients via compassionate access or early-phase clinical trials. Though our RPPA data and 9:1 male-to-female ratio hinted that DSRCT is an AR-driven malignancy, to prove this explicitly we proposed several criteria, akin to Koch's postulates: (a) tumors must adequately express AR, 
bioRxiv preprint doi: https://doi.org/10.1101/2022.01.20.477146; this version posted January 22, 2022. The copyright holder for this preprint (which was not certified by peer review) is the author/funder, who has granted bioRxiv a license to display the preprint in perpetuity. It is made available under aCC-BY-NC-ND 4.0 International license.

(b) DHT must stimulate DSRCT cell proliferation, and (c) AR antagonists should curtail the tumor-promoting effects of androgen stimulation. The inclusion of mechanistic studies, including those directly tying AR to androgen response elements (AREs), lends further credibility that DSRCT is a second AR-driven malignancy.

To date, the first criterion - requiring AR expression - has been reported by two prior teams that recognized the striking predilection of DSRCT for young males ${ }^{15,51}$. As discussed briefly in the Introduction, Fine et al. evaluated protein expression of AR, c-Kit, EGFR, and other proteins by Western blot and IHC, scored using a 5point scale that ranged between 0 (no staining) to 4+ (highly positive) ${ }^{15}$. Ten of twenty-seven (37\%) DSRCT patients in their case series scored 2+ or higher, but we highlight that fifteen demonstrated no AR expression (Figure 2), which suggests prospective studies may wish to stratify for response by AR-status to determine whether AR expression correlates with therapeutic efficacy. A more recent study published in 2018 by Bulbul et al. at U.C.S.D., used IHC and next-generation sequencing on tumors from thirty-five DSRCT patients $(86 \%$ who were males); $59 \%$ were AR-positive using a dichotomous cut-off that required $\geq 1+$ staining in $\geq 10 \%$ of the cells ${ }^{51}$. In the present study, we report the most extensive series of DSRCT patients to have undergone protein and transcriptomic profiling. Though enriched in oncoproteins, our RPPA array ranked AR as the most differentially expressed protein compared to ES, its closest molecular sarcoma subtype (Figure 1). Our subsequent confirmation of the RPPA results by Western blot, and later semi-quantitative analysis by IHC, is in agreement with earlier reports and appears to substantiate AR as a bona fide target in DSRCT.

Meeting the second of Koch's postulates, a 72-hour cell proliferation assay demonstrated a significant increase in JN-DSRCT cell proliferation following exposure to physiological levels of DHT (Figure 3A), though lower than LNCaP PC cells. As one would expect in androgen-sensitive cells, DHT also promoted the nuclear shuttling of AR into the nucleus where it would function as a transcriptional regulator of its target genes (Figure $3 C \& 3 D$ ). As our results rely upon data from a single cell line (the only one in existence at the time), we remain vigilant to avoid over-interpreting them. Nevertheless, our results echo similar findings by Fine et al., where they reported a DHT-induced 2-fold increase in cell proliferation in a transient DSRCT cell line obtained from ascitic fluid $^{18}$.

Fulfilling the third requirement that defines an AR-driven malignancy, our team again bolsters the work by Fine et al., which had taken a prescient step more than a decade ago to evaluate $C A B$ - in that case using Lupron and bicalutamide in six DSRCT patients that were AR-positive $(3+\text { or } 4+\text { by } \mathrm{IHC})^{18}$. Interestingly, in their limited pilot trial, non-castrate level baseline testosterone levels were associated with modest responses lasting 3-4 months. Admittedly, having tested several DSRCT patients with the same drug combination between $2006-$ 2015, well before the advent of modern-day androgen deprivation therapies (ADT) such as abiraterone and enzalutamide, our team observed limited clinical benefits lasting $<3$ months. Our renewed enthusiasm for AR targeting in DSRCT stemmed from the RPPA expression results, accompanied by the in vitro DHT stimulation studies and in vivo data using enzalutamide and the AR-ASO (Figure 4).

In preparation for early-phase clinical trials now in development, our work takes the first step to advance our mechanistic understanding of AR signaling in DSRCT. As one of several steroid and nuclear hormone superfamily receptors that include the glucocorticoid receptor (GR), mineralocorticoid receptor (MR), progesterone and estrogen receptors (PR \& ER), and the vitamin D receptor (VDR), AR retains a conserved 66amino acid DNA-binding domain (DBD) able to join two (5'-AGAACA-3') hexameric half-sites arranged as an inverted palindrome spaced 3-b.p. apart (IR3). Due to differences in local steroid metabolism, ligand abundance, chromatin accessibility, and cofactor occupancy, the DNA binding pattern of AR varies significantly in PC compared to other tissues ${ }^{52}$. Interestingly, among the pioneer factors that govern the lineage-specific binding of AR to specific genomic loci in $\mathrm{PC}^{53-55}$, and that control AR-mediated transcriptional regulation of prostate genes (such as PSA) ${ }^{56}$, FOXA1 was the most enriched MOTIF in JN-DSRCT cells (Figure 6C). Shared activation of the androgen signaling cascade in DSRCT and PC may explain the close transcriptomic clustering observed in Fig. 2E. Despite their similarities, ChIP-seq also identified notable differences in AR's epigenetic regulation at enhancer (Figure 7) and super-enhancer (Figure S9C) binding sites. 
Though the subject of future research, we suspect the heterotypic loss of WT1 or aberrant EWS-WT1 FP may recruit a specific set of chromatin modifiers at binding sites that differ from PC. Others have performed ChIP-seq in DSRCT patient specimens using WT1-specific antibodies, but the Santa Cruz antibody used in that publication $^{57}$ has been discontinued. Lacking suitable ChIP-seq validated WT1-specific antibodies ATAC-seq might be used before and after WT1 RNA silencing, though interpretation of that experiment wouldn't be as straightforward given the absence of selective antagonism of WT1 or EWS-WT1.

Interestingly, as occurs in castration-resistant $\mathrm{PC}^{11,58,59}$, our pharmacodynamic studies revealed an inverse relationship between AR and the Akt/PI3K/mTOR pathway. With numerous inhibitors of PI3K and mTOR already FDA-approved, an obvious next step would be to investigate whether co-targeting AR and either PI3K or mTOR results in synergistic anti-cancer activity. Though not explored in the present study, the estrogen receptor (ER) was also highly expressed following AR-ASO treatment. Given the shared binding DNA motifs that ER, AR, and other steroid hormone receptors have in common, this observation suggests that ER-targeted drugs might prove useful for patients with castrate-resistant DSRCT and, plausibly, the small minority of women that acquire this rare cancer type. Of course, further research in required to determine how AR and ER pathway switching affects tumor growth and survival, both in DSRCT and other hormonally-driven malignancies ${ }^{60}$.

Collectively, though morphologically and phenotypically distinct from PC, our data suggest that DSRCT is a second androgen-stimulated malignancy (third, if one considers the AR-positive molecular subset of triplenegative breast cancer). Shared dependence upon AR for tumor growth and survival provides an exciting opportunity to study AR signaling in a different cancer type and within a younger DSRCT-stricken patient population. Preclinical data using enzalutamide and AR-ASO raises the tantalizing possibility that AR-targeted drugs used for PC may also find utility to combat DSRCT.

\section{PATIENTS AND METHODS}

\section{Patients}

The collection of DSRCT tumor patients was approved by the Institutional Review Board of MDACC under the LAB08-0151 and LAB04-0890 protocols and conducted in compliance with the principals of the Declaration of Helsinki. The charts and electronic medical records of patients with a confirmed diagnosis of DSRCT were included for analysis and archived at the MDACC biospecimen bank or the collaborator PIs laboratories. We identified 60 DSRCT patients treated at MDACC from 1990 to 2019 to generate a TMA. Also, we collected 16 DSRCT and 6 Ewing sarcoma (ES) fresh frozen tumors, all of them were profiled by RPPA. Specialist pathologists used clinical information, immunohistochemistry, and cytogenic analysis for the EWSR1-WT1 or EWSR1-FLI1 fusions to confirm the DSRCT or ES diagnoses. Blood samples (EDTA) of $5 \mathrm{ml}$ were collected from 17 DSRCT patients and 3 Ewing sarcoma patients (ES) to remove serum and assay PSA.

\section{RPPA and Western blot analyses}

The available snap-frozen DSRCT $(n=16)$ and ES $(n=6)$ specimens collected during a core-needle biopsy or surgical debulking procedures using clinical protocols approved by MDACC's Institutional Review Board and specimens of normal-appearing mesenteric tissue adjacent to DSRCT obtained at the time of surgical debulking $(n=8)$ were used for the proteomic analysis (Supplemental Table 4: Demographic information of DSRCT and ES patients). Lysates were created, protein concentrations were determined, and individual protein expression was measured using a well-validated reverse-phase protein array (RPPA) and Western blot (WB) technologies as previously described ${ }^{61-63}$. AR protein detection was performed using the CST antibody (\#5153). Additional details about RPPA and WB analyses and normalized data are provided in the Supplementary Methods and Supplemental Table 5.

\section{RNA Sequencing, gene expression analysis, and fusion detection}

Total RNA from primary tumor samples was extracted and libraries made from cDNA using the NuGEN Ovation Ultralow Library System V2 (San Carlos, CA). RNA sequencing reads of the samples were mapped to the hg19 
reference genome using the STAR aligner ${ }^{64}$. For calculation of gene expression, each gene's raw count data were first obtained using HTSeq ${ }^{65}$, and are normalized by scaling the library size using calcNormFactors in the edgeR package ${ }^{66}$. Then, Voom transformation was applied to normalized counts and a linear model fit to the data for differential expression analysis using the Limma package ${ }^{67}$. Pathway analyses of differentially expressed genes between two sample clusters were performed using Gene Set Enrichment Analysis (GSEA) ${ }^{68}$. Fusion transcripts were detected from RNA-seq data using MapSplice ${ }^{69}$.

\section{TMA Preparation and Immunohistochemistry Analyses}

A tissue microarray (TMA) was constructed from archival surgical pathology materials comprising 60 formalinfixed, paraffin-embedded tissues from 60 DSRCT patients. Areas of the viable tumor were selected by pathologist review of whole slide H\&E-stained sections. Selected areas were punched and transferred, in duplicate, to a recipient block using an ATA-100 Advanced Tissue Arrayer (Chemicon International). All human specimens were utilized under an Institutional Review Board-approved research protocol (LAB04-0890) allowing for the retrospective sampling and analysis of existing archival materials collected in the course of standard patient care. Immunohistochemical studies were performed using an autostainer (Bond-Max; Leica Microsystems, Buffalo Grove, IL, USA) with anti-AR (1:30; clone AR441, Dako\#M3562) antibody. Additional details about TMA slides preparation and IHC analyses are provided in the Supplementary Methods.

\section{WST1 Cell Proliferation Assays}

The JN-DSRCT, LNCaP, and TC71 cells tested for their proliferation capacity In vitro using a colorimetric assay in 96-Well plates with WST-1 reagent (Roche). The cells seeded at 3000 cells/well in triplicates with 10\% FBS DMEM (JN-DSRCT) or RPMI (TC71 and LNCaP) complete media. Additional details about WST1 cell proliferation assays are provided in the Supplementary Methods.

\section{Immunostaining of JN-DSRCT cells and xenograft animal tumors}

JN-DSRCT cell line exhibiting a pathognomonic $\mathrm{t}(11 ; 22)(\mathrm{p} 13 ; \mathrm{q} 12)$ translocation was generously provided from Dr. M Kikuchi's laboratory (Fukuoka University, Fukuoka, Japan). In addition, PC3, LnCaP, and TC71 cell lines are provided by the MDA cell lines core facility. All available cell lines in Dr. Ludwig's lab are registered within the MDA characterized cell line core (CCLC). Each cell line identity is validated twice per year in MDA CCLC using short-tandem repeat (STR) fingerprinting with an AmpFLSTR Identifier kit. Furthermore, according to the manufacturer's protocol, all our cell lines are tested twice per year for mycoplasma contamination using the MycoAlert Detection Kit (Lonza Group Ltd.). Additionally, cell lines are sent for 3rd-party mycoplasma testing using a sensitive PCR testing approach any time a collection of cells are cryopreserved.

Monolayer JN-DSRCT cell culture in 8 chamber slides were fixed for $10 \mathrm{~min}$ at room temperature with $4 \%$ paraformaldehyde in phosphate-buffered saline (PBS). The primary JN-DSRCT xenograft and PDX tumors were harvested, fixed in 10\% formalin, embedded in paraffin (formalin-fixed, paraffin-embedded: FFPE), and then sliced in $5 \mu \mathrm{m}$ sections before processing them for antigen retrieval using $0.1 \mathrm{M}$ citrate buffer for 20 minutes and in a vegetable steamer. Altogether, monolayer and primary tumor slides were permeabilized and blocked with superblock buffer (Thermo Fisher Scientific, \#37535) for 1 hour at room temperature. Slides were then incubated consecutively with primary antibodies to AR (Cell Signaling Technologies, \#5153), (overnight at $4^{\circ} \mathrm{C}$ ) and Alexa Fluor 488-labeled Goat-anti Rabbit (Thermo Fisher Scientific, \#A11037) (for $1 \mathrm{hr}$ at room temperature). The nuclei were visualized using Hoechst (Thermo Fisher Scientific, \#H357), and the immunofluorescence was acquired after subtracting the background intensities using the Nikon A1-Rsi confocal microscope (Nikon). Fluorescentdetection of proteins in the nuclei and cytosolic regions was quantified using the Imaris software (Bitplane) and its Cell module that use validated algorithms to define the segmentation by permitting the recognition of selected protein fluorescence in both nuclear and cytosolic regions.

\section{Generation of DSRCT Xenograft/PDX mouse models and Drug Evaluation}

All experiments were conducted per protocols and conditions approved by the University of Texas MD Anderson Cancer Center (MDACC; Houston, TX) Institutional Animal Care and Use Committee (eACUF Protocols 
\#00000712-RN03). Male NOD (SCID)-IL-2Rg ${ }^{\text {null }}$ mice (The Jackson Laboratory; Farmington, CT) were subcutaneously injected with JN-DSRCT cells $\left(5 \times 10^{6}\right.$ cells/animal) or received PDX explants $(2 \mathrm{~mm})$ to generate DSRCT xenograft and PDX mouse models. The histologic and genetic analyses of DSRCT patient and PDX tumors are available on Supplemental Figure 12. All mice were maintained under barrier conditions and treated using protocols approved by The University of Texas MD Anderson Cancer Center's Institutional Animal Care and Use Committee. Once their tumors reached a volume of $150 \mathrm{~mm}^{3}, 5$ mice per group received enzalutamide (25 mg/kg IP daily, 5 times per week), or AR ASOs ( 25 or 50 mg/kg subcutaneously daily, 5 times per week), or control ASOs (50 mg/kg subcutaneously daily, 5 times per week), or a placebo control (sterile vehicle buffer). Tumor volumes were measured using digital calipers at study initiation and 2-5 times per week after that for up to 85 days, or until their tumors reached $1500 \mathrm{~mm}^{3}$, whichever came first. A Kaplan-Meier analysis was performed to assess drug efficacy. Statistical analyses between control and treated group or between different treated groups were performed with the log-rank (Mantel-Cox) test using Graph-Pad Prism 8.0.

\section{Mass spectroscopy based determination of intratumoral hormone levels}

Testosterone and corticosterone quantification were determined using Agilent's Infinity II UHPLC in line with a 6495 triple quadrupole mass spectrometer and MassHunter workstation software (8.0.8.23.5). Briefly, DSRCT xenograft and PDX samples were homogenized using water containing internal standard (Cerilliant, T070) extracted with tert-butyl methyl ether (Sigma 34875), dried under nitrogen, and derivatized using hydroxylamine hydrochloride (Sigma 431362). The recovered ketoxime steroids were reconstituted in methanol/water (1:1 v/v) and injected into the Infinity II UHPLC. Ketoxime steroids were separated using a Chromolith reverse phase column (RP-18 endcapped 100-2mm, Sigma 152006) and introduced into a JetStream source (Agilent) for triple quadrupole analysis. Data were analyzed and quantified using MassHunter software (Agilent) ${ }^{39,40}$.

\section{ChIP-Seq Assays}

Chromatin immunoprecipitation was performed as described earlier ${ }^{70}$ with optimized shearing conditions and minor modifications for JN-DSRCT cells. The antibodies used were: H3K27ac (Abcam ab4729) and AR (CST\#5153). Briefly, 3 million cells per sample were cross-linked using $1 \%$ formaldehyde for 10 min at $37^{\circ} \mathrm{C}$. After quenching with $150 \mathrm{mM}$ glycine for $5 \mathrm{~min}$ at $37^{\circ} \mathrm{C}$, cells were washed twice with ice-cold PBS and frozen at $-80{ }^{\circ} \mathrm{C}$ for further processing. Later, cells were thawed on ice and lysed with ChIP harvest buffer (12 mM Tris$\mathrm{HCl}, 0.1 \times \mathrm{PBS}, 6 \mathrm{mM}$ EDTA, $0.5 \%$ sodium dodecyl sulfate [SDS]) for $30 \mathrm{~min}$ on ice. Lysed cells were sonicated with Bioruptor (Diagenode) to obtain chromatin fragment. Antibody-dynabead mixtures were incubated for $1 \mathrm{hr}$ at $4{ }^{\circ} \mathrm{C}$ and cellular extracts were then incubated overnight with these mixtures. After overnight incubation, immune complexes were washed five times with RIPA buffer, twice with RIPA-500 (RIPA with $500 \mathrm{mM} \mathrm{NaCl}$ ) and twice with $\mathrm{LiCl}$ wash buffer $(10 \mathrm{mM}$ Tris- $\mathrm{HCl}$ pH8.0, $1 \mathrm{mM}$ EDTA pH8.0, $250 \mathrm{mM} \mathrm{LiCl,} \mathrm{0.5 \%} \mathrm{NP-40,} \mathrm{0.1 \%}$ deoxycholate). For reverse-crosslinking and elution, immune complexes were incubated overnight at $65{ }^{\circ} \mathrm{C}$ in elution buffer (10 mM Tris- $\mathrm{HCl}$ pH8.0, $5 \mathrm{mM}$ EDTA, $300 \mathrm{mM} \mathrm{NaCl}, 0.5 \%$ SDS). Eluted DNA was then treated with proteinase $\mathrm{K}(20 \mathrm{mg} / \mathrm{ml})$ and RNase A and DNA clean-up was done using SPRI beads (Beck- man-Coulter). ChIP libraries were amplified and barcoded with use of the NEBNext@ Ultra ${ }^{\mathrm{TM}}$ II DNA library preparation kit (New England Biolabs). After library amplification, DNA fragments were size-selected (200 - 500 bp) using AMPure XP beads (Beckman Coulter) and assessed using high sensitivity D1000 screen tape on the Bioanalyzer (Agilent Technologies). Libraries were multiplexed together and sequenced in HiSeq2000 (Illumina).

\section{ChIP-seq Data Processing}

ChIP-seq data were quality controlled and processed by pyflow-ChIPseq ${ }^{71}$, a snakemake ${ }^{72}$ based ChIP-seq pipeline. Briefly, raw reads were mapped by bowtie $1^{73}$ to $\mathrm{hg} 19$. Duplicated reads were removed, and only uniquely mapped reads were retained. RPKM normalized bigwigs were generated by deep tools ${ }^{74}$, and tracks were visualized with IGV ${ }^{75}$. Peaks were called using macs $1.4^{76}$ with a $\mathrm{p}$-value of $1 \mathrm{e}-9$ for H3K27ac and $1 \mathrm{e}-7$ for AR. Heatmaps were generated using $R$ package EnrichedHeatmap. ChIP-seq peaks were annotated with the 
nearest genes using ChIPseeker ${ }^{77}$. Super-enhancers were identified using ROSE $^{78}$ based on H3K27ac ChIPseq data.

\section{Differential Peaks Analysis}

To identify variable AR or enhancer domains enriched in specific DSRCT samples, enhancer peaks that overlap with $2.5 \mathrm{~kb}$ upstream and $2.5 \mathrm{~kb}$ downstream of any known TSSs were removed. The unique and shared peaks within multiple groups were identified by Intervene ${ }^{79}$. The peaks were annotated with ChIPseeker R package ${ }^{77}$, using addFlankGenelnfo function for enhancers.

\section{Identification of AR and Enhancer Associated Pathways}

Differential AR binding sites and enhancers associated genes in each sample were imported into the ClusterProfiler ${ }^{80}$ for pathway analysis, restricted to GO, KEGG, Hallmark, and WiKi gene sets. The Enrichplot package ${ }^{81}$ was used to generate dot plot and bar plot for gene sets enriched with a false discovery rate (FDR) cut-off of $<0.05$.

\section{Enrichment of Motifs in AR-Specific Peaks}

To identify the motifs over-represented within AR-specific peak sets, we used the HOMER motif database and the coordinates of AR-specific peak sets ${ }^{82}$. 


\section{FIGURE LEGENDS}

Fig 1. Proteomic comparison of DSRCT and ES. A) The protein lysates from DSRCT (red) and ES (blue) were subjected to RPPA analysis for 151 proteins and phosphoproteins (red, increased signal; green, decreased signal). Unsupervised double-hierarchical clustering using the Pearson correlation distance metric between proteins (rows) and Centroid linkage (a clustering method) separated the 22 samples into two groups by tumor type (columns). Of the 22 proteins, 8 had expression that differed significantly between ES and DSRCT ( $p \leq 0.05$; fold-change $\geq 2$ ). B) The mean expression intensity values of the 8 proteins associated with DSRCT or ES and their statistical significance after normalization for global protein expression by median centering across 151 antibodies in the RPPA panel. C) Western blotting was used to validate the proteins identified by RPPA as being differentially expressed between DSRCT and ES. D) Normalized protein expression is relative to $\beta$-actin.

Fig 2. DSRCT TMA and frozen specimen profiling for AR and PSA expression. A) A histogram showing the AR IHC expression levels of 60 human DSRCT tumors grouped by intensity (low, moderate, and high). The demographic data, including the corresponding gender (Red: male or Green: female), the age at diagnosis, and the pre/post-chemotherapy treatment to the surgery of each primary or metastatic resected tumor patient, are displayed at the left of each histogram. B) AR expression level interpretation on DSRCT TMA IHC-stain and percentage scoring of tumoral labeling (Positive $(>50 \%)$, Low positive (10-50\%), Focal $(1-10 \%)$, and Negative $(0-1 \%))$. C) Western blotting analyses of AR expression in 11 DSRCT snap-frozen primary tumors. AR expression: $P=$ positive, $N=$ negative, or $M=$ moderate. $D)$ Relative $A R$ levels across samples shown in $C$ ). Bars show mean \pm SD. E) The principal components analysis plot performed on gene expression from prostate cancer (PC), DSRCT, and additional type of sarcomas samples. F) Boxplot for the AR gene expression level across DSRCT, prostate cancer, and four other sarcoma types. The Wilcoxon rank-sum test performed to compare the AR levels between DSRCT and each of the other cancer types. Chondro=chondrosarcoma; Osteo=osteosarcoma; WDLPS=well-differentiated liposarcoma, and DDLPS=dedifferentiated liposarcoma.

Fig 3. In vitro Stimulation and inhibition of DSRCT proliferation via AR. A) JN-DSRCT, TC71, and LNCaP Cell proliferation assays after treating them with an AR agonist hormone, dihydrotestosterone (DHT) in a dosedependent manner. B) Profiling JN-DSRCT, TC71, and LNCaP cells for their AR expression by western blotting and histogram presentation of relative AR levels across each cell line. C) Profiling of JN-DSRCT cells for AR protein expression (green) by immunofluorescence analysis with DAPI-labeled nuclei (blue), D) and quantitative scatter plot representation of the ratio Nuclear/Cytoplasmic AR mean intensity reported within a single cell at 0 , 6,18 , and 18 hours of DHT post-treatment. Bars represent standard deviations. E) JN-DSRCT cells are relatively less sensitive to enzalutamide than, F) AR antisense oligonucleotides treatment, as shown by the in vitro WST1Proliferation cell-based assay. G) Western blot analysis of AR expression in JN-DSRCT cells untreated or after Control-ASO and AR-ASO treatments. Histogram presentation of relative AR levels across each cell line after GAPDH normalization.

Fig 4. Preclinical efficacy of AR antisense-based therapy for the treatment of DSRCT. A-B) Therapeutic effect of AR blockade in JN-DSRCT xenografts done in three replicates. Tumor-bearing mice volumes, and survival were reported after been treated with the enzalutamide $(25 \mathrm{mmg} / \mathrm{kg}$, orange), the AR-ASO $(25 \mathrm{mg} / \mathrm{kg}$, regular red; $50 \mathrm{mg} / \mathrm{kg}$, dotted red), control ASO (gray), and placebo treatment (black). A) The left panel shows the smoothed grouped median relative tumor volumes in these groups of mice. The $P$ values for differences between the treated and control mice were performed with the log-rank (Mantel-Cox) test. B) The right panel shows the survival Kaplan-Meier curves of each treated group of mice. C-D) Therapeutic effect of AR antisense blockade in JN-DSRCT xenografts. C) Tumor-bearing mice volumes were reported through smoothed grouped median relative tumor volumes after been treated the mice with the AR-ASO $(50 \mathrm{mg} / \mathrm{kg}$, red), control ASO (gray), and placebo treatment (black). D) Kaplan-Meier curves show mouse survival after drug treatment. The $P$ values 
for differences between the treated and control mice were performed with the log-rank (Mantel-Cox) test. E-F) Therapeutic effect of AR antisense blockade in DSRCT PDX1 mice. E) The smoothed grouped median relative tumor volumes are shown after the mice been treated with the AR-ASO (50mg/kg, red), control ASO (gray), and placebo treatment (black). F) Kaplan-Meier curves indicate the survival rate after drug treatment. The $P$ values for differences between the treated and control mice were performed with the log-rank (Mantel-Cox) test.

Fig 5. Proteomic evaluation of AR expression in JN-DSRCT and PDX tumors after AR-based antisense therapy. A) The principal components analysis plot and reverse-phase protein lysate array (RPPA) evaluations of JN-DSRCT and PDX tumors after therapies, separated the 32 samples into four groups and identified 37 proteins statistically significantly associated with the treatment at a false discovery rate (FDR) of 0.05 . B) Immunoblotting evaluation of JN-DSRCT xenograft and PDX-DSRCT tumors after AR-ASO treatment. C) AR normalization relative to GAPDH within the preclinical tumor samples. AR biomarker was significantly reduced in mice treated with AR-ASO compared to the control ASO group $(p=0.01)$. D) Representative AR immunofluorescence confocal microscopy quantification of the preclinical JN-DSRCT and PDX tumor samples, within the single cell or, E) the averaged treated samples (placebo, control ASO, and AR-ASO). Bars represent standard deviations. F) Immunohistochemical evaluation images of preclinical JN-DSRCT and PDX1 tumor samples. IHC stains for AR in primary tumors of JN-DSRCT and PDX DSRCT mice after treatment with ARASO, control ASO, and placebo. $100 \mu \mathrm{m}$ scale bars are shown. G) Representative IHC AR mean intensity quantification of the preclinical JN-DSRCT and PDX tumor samples, within the single cell or, $\mathrm{H}$ ) the averaged treated samples (placebo, control ASO, and AR-ASO). Bars represent standard deviations. All tumors analyzed by PPPA were collected at tumor progression or the experiment's conclusion, except for the AR-ASO PD group, which was collected 10 days after initiating therapy with pharmacodynamic analysis.

Fig 6. AR binding in JN-DSRCT cells. A) Heatmaps (left panels) and average intensity curves (right panels) of ChIP-seq reads (RPKM; reads per kilobase of transcript per million mapped reads) for AR binding regions. AR binding sites are shown in a 10-kb window (centered on the middle of the binding site) in Control ASO, AR-ASO, $\mathrm{DHT}+$ Control ASO, and DHT + AR ASO samples. B) Venn diagram showing the overlap of all AR peaks between Control ASO, DHT + Control ASO, and DHT + AR-ASO samples to identify the AR-unique or shared binding sites. C) List of enriched transcription factor (TF) motifs in AR-specific binding sites. Motifs are identified using HOMER. D) Dot plot showing significantly enriched pathways for AR specific binding sites. Dot size represents gene ratio, and colors represent adjusted p-values. E) IGV images showing enrichment of AR peaks around WT1, SOX2, CTNNB1, GATA6, FOXF1, and GLI2 genes using aggregate ChIP-seq profiles of Control ASO, DHT + Control ASO and DHT + AR-ASO samples.

Fig 7. Enhancer reprogramming by AR in JN-DSRCT cells. A) Heatmaps (left panels) and average intensity curves (right panels) of ChIP-seq reads (RPKM; reads per kilobase of transcript per million mapped reads) for typical enhancer regions. Enhancer regions are shown in a 10-kb window (centered on the middle of the binding site) in Control ASO, AR-ASO, DHT + Control ASO, and DHT + AR ASO samples. B) Venn diagram showing the overlap of all enhancer peaks between Control ASO, AR-ASO, DHT + Control ASO, and DHT + AR ASO samples to identify the AR-unique or shared enhancer reprogramming. C) Venn diagram showing the overlap of annotated genes for AR specific gained enhancer peaks and upregulated gene list for DSRCT tumors vs. other sarcoma tumors to identify the AR-unique enhancer reprogramming associated transcription upregulation. D) Bar plot showing significantly enriched pathways for AR specific enhancer reprogramming associated transcription upregulation. Bar length represents gene numbers, and colors represent adjusted $p$-values. E) IGV images showing enrichment of H3K27Ac peaks around AGRE2, AXIN2, CDK6, and MYH10 genes using aggregate ChIP-seq profiles of Control ASO, DHT + Control ASO, and DHT + AR-ASO samples. 


\section{Data availability}

All ChIP-seq data are available at GEO accession number GSE151380. All codes used to generate the ChIPseq data are available at https://github.com/crazyhottommy/pyflow-ChIPseq. RNA-seq data for DSRCT are under EGAS00001004575, liposarcoma under EGAS00001002807, osteosarcoma under EGAS00001003247, chondrosarcoma under EGAS00001004585, and prostate cancer through Subudhi et al ${ }^{83}$.

RPPA (Fig. 1A-B):

RNA-Seq for DSRCT (Fig 2E\&F):

RNA-Seq for Liposarcoma (Fig 2E\&F):

RNA-Seq for Osteosarcoma (Fig 2E\&F):

RNA-Seq for Chondrosarcoma (Fig 2E\&F):

RNA-Seq for Prostate Cancer (Fig 2E\&F):

RPPA, Figure 5A:

AR-ChIP-seq (Fig. 6):

H3K27Ac ChIP-seq (Fig. 7):

RPPA, Supplemental (Fig. 1A-B):

RPPA, Supplemental Figure 6F-G

AR-ChIP-seq, Supplemental Fig. 7:

H3K27Ac ChIP-seq, Supplemental Fig. 8:
GEO (Accession \#: GSE108687)

EGAS00001004575

EGAS00001002807

EGAS00001003247

EGAS00001004585

Subudhi et al. ${ }^{83}$

GEO (Accession \#: GSE178406)

GEO (Accession \#: GSE151380)

GEO (Accession \#: GSE151380)

GEO (Accession: GSE108687)

GEO (Accession \#: GSE178406)

GEO (Accession \#: GSE151380)

GEO (Accession \#: GSE151380) 


\section{REFERENCES}

1 Honore, C. et al. Abdominal Desmoplastic Small Round Cell Tumor: Multimodal Treatment Combining Chemotherapy, Surgery, and Radiotherapy is the Best Option. Ann Surg Oncol, doi:10.1245/s10434-0144123-6 (2014).

2 Chang, F. Desmoplastic small round cell tumors: cytologic, histologic, and immunohistochemical features. Archives of pathology \& laboratory medicine 130, 728-732, doi:10.1043/15432165(2006)130[728:DSRCTC]2.0.CO;2 (2006).

3 Gerald, W. L., Rosai, J. \& Ladanyi, M. Characterization of the genomic breakpoint and chimeric transcripts in the EWS-WT1 gene fusion of desmoplastic small round cell tumor. Proc Natl Acad Sci US A 92, 1028-1032 (1995).

4 Gerald, W. L. et al. Clinical, pathologic, and molecular spectrum of tumors associated with $\mathrm{t}(11 ; 22)(\mathrm{p} 13 ; \mathrm{q} 12)$ : desmoplastic small round-cell tumor and its variants. J Clin Oncol 16, 3028-3036 (1998).

Lettieri, C. K., Garcia-Filion, P. \& Hingorani, P. Incidence and outcomes of desmoplastic small round cell tumor: results from the surveillance, epidemiology, and end results database. Journal of cancer epidemiology 2014, 680126, doi:10.1155/2014/680126 (2014).

Gerald, W. L. \& Rosai, J. Case 2. Desmoplastic small cell tumor with divergent differentiation. Pediatric pathology / affiliated with the International Paediatric Pathology Association 9, 177-183 (1989).

Shen, W. P., Towne, B. \& Zadeh, T. M. Cytogenetic abnormalities in an intraabdominal desmoplastic small cell tumor. Cancer Genet Cytogenet 64, 189-191, doi:10.1016/0165-4608(92)90355-c (1992).

Ladanyi, M. \& Gerald, W. Fusion of the EWS and WT1 genes in the desmoplastic small round cell tumor. Cancer Res 54, 2837-2840 (1994).

Sawyer, J. R., Tryka, A. F. \& Lewis, J. M. A novel reciprocal chromosome translocation t(11;22)(p13;q12) in an intraabdominal desmoplastic small round-cell tumor. Am J Surg Pathol 16, 411-416, doi:10.1097/00000478-199204000-00010 (1992).

Gedminas, J. M. et al. Desmoplastic small round cell tumor is dependent on the EWS-WT1 transcription factor. Oncogenesis 9, 41, doi:10.1038/s41389-020-0224-1 (2020).

1 Thomas, C. et al. Synergistic targeting of PI3K/AKT pathway and androgen receptor axis significantly delays castration-resistant prostate cancer progression in vivo. Mol Cancer Ther 12, 2342-2355, doi:10.1158/1535-7163.MCT-13-0032 (2013).

2 Chan, J. J., Tan, T. J. Y. \& Dent, R. A. Novel therapeutic avenues in triple-negative breast cancer: PI3K/AKT inhibition, androgen receptor blockade, and beyond. Ther Adv Med Oncol 11, 1758835919880429, doi:10.1177/1758835919880429 (2019).

13 Subbiah, V. et al. Multimodality Treatment of Desmoplastic Small Round Cell Tumor: Chemotherapy and Complete Cytoreductive Surgery Improve Patient Survival. Clin Cancer Res 24, 4865-4873, doi:10.1158/1078-0432.CCR-18-0202 (2018).

14 Mora, J. et al. Desmoplastic small round cell tumor 20 years after its discovery. Future oncology 11, 10711081, doi:10.2217/fon.15.32 (2015).

15 Fine, R. L. et al. Androgen and c-Kit receptors in desmoplastic small round cell tumors resistant to chemotherapy: novel targets for therapy. Cancer chemotherapy and pharmacology 59, 429-437, doi:10.1007/s00280-006-0280-z (2007).

16 Efstathiou, E. et al. Molecular characterization of enzalutamide-treated bone metastatic castrationresistant prostate cancer. Eur Urol 67, 53-60, doi:10.1016/j.eururo.2014.05.005 (2015).

17 Labrie, M. et al. Using Reverse Phase Protein Array (RPPA) to Identify and Target Adaptive Resistance. Adv Exp Med Biol 1188, 251-266, doi:10.1007/978-981-32-9755-5_14 (2019).

18 Akbani, R. et al. Realizing the promise of reverse phase protein arrays for clinical, translational, and basic research: a workshop report: the RPPA (Reverse Phase Protein Array) society. Mol Cell Proteomics 13, 1625-1643, doi:10.1074/mcp.O113.034918 (2014). 
bioRxiv preprint doi: https://doi.org/10.1101/2022.01.20.477146; this version posted January $22,2022$. The copyright holder for this preprint

(which was not certified by peer review) is the author/funder, who has granted bioRxiv a license to display the preprint in perpetuity. It is made available under aCC-BY-NC-ND 4.0 International license.

19 Mitra, R., Mueller, P., Ji, Y., Mills, G. \& Lu, Y. Sparse Bayesian Graphical Models for RPPA Time Course Data. IEEE Int Workshop Genomic Signal Process Stat 2012, 113-117, doi:10.1109/GENSIPS.2012.6507742 (2012).

20 Carceles-Cordon, M. et al. Cellular rewiring in lethal prostate cancer: the architect of drug resistance. Nat Rev Urol, doi:10.1038/s41585-020-0298-8 (2020).

21 Wang, G., Zhao, D., Spring, D. J. \& DePinho, R. A. Genetics and biology of prostate cancer. Genes Dev 32, 1105-1140, doi:10.1101/gad.315739.118 (2018).

22 Kregel, S. et al. Differential modulation of the androgen receptor for prostate cancer therapy depends on the DNA response element. Nucleic Acids Res, doi:10.1093/nar/gkaa178 (2020).

23 Kregel, S. et al. Androgen receptor degraders overcome common resistance mechanisms developed during prostate cancer treatment. Neoplasia 22, 111-119, doi:10.1016/j.neo.2019.12.003 (2020).

24 Holly, S. P., Larson, M. K. \& Parise, L. V. Multiple roles of integrins in cell motility. Exp Cell Res 261, 69-74, doi:10.1006/excr.2000.5040 (2000).

25 Kechagia, J. Z., Ivaska, J. \& Roca-Cusachs, P. Integrins as biomechanical sensors of the microenvironment. Nat Rev Mol Cell Biol 20, 457-473, doi:10.1038/s41580-019-0134-2 (2019).

26 Hornebeck, W., Emonard, H., Monboisse, J. C. \& Bellon, G. Matrix-directed regulation of pericellular proteolysis and tumor progression. Semin Cancer Biol 12, 231-241, doi:10.1016/s1044-579x(02)00026-3 (2002).

27 Antonarakis, E. S. et al. Androgen receptor splice variant, AR-V7, and resistance to enzalutamide and abiraterone in men with metastatic castration-resistant prostate cancer (mCRPC). Journal of Clinical Oncology 32, doi:DOI 10.1200/jco.2014.32.15_suppl.5001 (2014).

28 Paschalis, A. et al. Alternative splicing in prostate cancer. Nature reviews. Clinical oncology 15, 663-675, doi:10.1038/s41571-018-0085-0 (2018).

29 Qu, Y. Y. et al. Constitutively Active AR-V7 Plays an Essential Role in the Development and Progression of Castration-Resistant Prostate Cancer. Sci Rep-Uk 5, doi:ARTN 7654

10.1038/srep07654 (2015).

30 Lamb, L. E., Zarif, J. C. \& Miranti, C. K. The androgen receptor induces integrin alpha6beta1 to promote prostate tumor cell survival via NF-kappaB and Bcl-xL Independently of PI3K signaling. Cancer Res 71, 2739-2749, doi:10.1158/0008-5472.CAN-10-2745 (2011).

31 Zarif, J. C., Lamb, L. E., Schulz, V. V., Nollet, E. A. \& Miranti, C. K. Androgen receptor non-nuclear regulation of prostate cancer cell invasion mediated by Src and matriptase. Oncotarget 6, 6862-6876, doi:10.18632/oncotarget.3119 (2015).

32 Taylor, B. S. et al. Integrative genomic profiling of human prostate cancer. Cancer Cell 18, 11-22, doi:10.1016/j.ccr.2010.05.026 (2010).

33 Dasgupta, S., Lonard, D. M. \& O'Malley, B. W. Nuclear receptor coactivators: master regulators of human health and disease. Annu Rev Med 65, 279-292, doi:10.1146/annurev-med-051812-145316 (2014).

34 Dasgupta, S. et al. Coactivator SRC-2-dependent metabolic reprogramming mediates prostate cancer survival and metastasis. $J$ Clin Invest 125, 1174-1188, doi:10.1172/JCI76029 (2015).

35 Foley, C. \& Mitsiades, N. Moving Beyond the Androgen Receptor (AR): Targeting AR-Interacting Proteins to Treat Prostate Cancer. Horm Cancer 7, 84-103, doi:10.1007/s12672-015-0239-9 (2016).

36 Agoulnik, I. U. et al. Role of SRC-1 in the promotion of prostate cancer cell growth and tumor progression. Cancer Res 65, 7959-7967, doi:10.1158/0008-5472.CAN-04-3541 (2005).

37 Yan, J. et al. Steroid receptor coactivator-3/AIB1 promotes cell migration and invasiveness through focal adhesion turnover and matrix metalloproteinase expression. Cancer Res 68, 5460-5468, doi:10.1158/0008-5472.CAN-08-0955 (2008).

38 Kemppainen, J. A., Lane, M. V., Sar, M. \& Wilson, E. M. Androgen receptor phosphorylation, turnover, nuclear transport, and transcriptional activation. Specificity for steroids and antihormones. $J$ Biol Chem 267, 968-974 (1992). 
39 Efstathiou, E. et al. Enzalutamide in Combination with Abiraterone Acetate in Bone Metastatic Castration-resistant Prostate Cancer Patients. Eur Urol Oncol 3, 119-127, doi:10.1016/j.euo.2019.01.008 (2020).

40 Maity, S. N. et al. Targeting of CYP17A1 Lyase by VT-464 Inhibits Adrenal and Intratumoral Androgen Biosynthesis and Tumor Growth of Castration Resistant Prostate Cancer. Sci Rep 6, 35354, doi:10.1038/srep35354 (2016).

41 Courtney, K. D., Corcoran, R. B. \& Engelman, J. A. The PI3K pathway as drug target in human cancer. J Clin Oncol 28, 1075-1083, doi:10.1200/JCO.2009.25.3641 (2010).

42 Carver, B. S. et al. Reciprocal feedback regulation of PI3K and androgen receptor signaling in PTENdeficient prostate cancer. Cancer Cell 19, 575-586, doi:10.1016/j.ccr.2011.04.008 (2011).

43 Gerhardt, J. et al. FOXA1 promotes tumor progression in prostate cancer and represents a novel hallmark of castration-resistant prostate cancer. The American journal of pathology 180, 848-861, doi:10.1016/j.ajpath.2011.10.021 (2012).

44 Takeda, D. Y. et al. A Somatically Acquired Enhancer of the Androgen Receptor Is a Noncoding Driver in Advanced Prostate Cancer. Cell 174, 422-432 e413, doi:10.1016/j.cell.2018.05.037 (2018).

45 Jin, L. et al. Therapeutic Targeting of the CBP/p300 Bromodomain Blocks the Growth of CastrationResistant Prostate Cancer. Cancer Res 77, 5564-5575, doi:10.1158/0008-5472.CAN-17-0314 (2017).

46 Bradner, J. E., Hnisz, D. \& Young, R. A. Transcriptional Addiction in Cancer. Cell 168, 629-643, doi:10.1016/j.cell.2016.12.013 (2017).

47 Huang, J. et al. Dissecting super-enhancer hierarchy based on chromatin interactions. Nat Commun 9 , 943, doi:10.1038/s41467-018-03279-9 (2018).

48 Khan, A. \& Zhang, X. Integrative modeling reveals key chromatin and sequence signatures predicting super-enhancers. Sci Rep 9, 2877, doi:10.1038/s41598-019-38979-9 (2019).

49 Menegaz, B. A. et al. Clinical Activity of Pazopanib in Patients with Advanced Desmoplastic Small Round Cell Tumor. Oncologist 23, 360-366, doi:10.1634/theoncologist.2017-0408 (2018).

50 Modak, S., Kramer, K., Gultekin, S. H., Guo, H. F. \& Cheung, N. K. Monoclonal antibody 8H9 targets a novel cell surface antigen expressed by a wide spectrum of human solid tumors. Cancer Res 61, 40484054 (2001).

51 Bulbul, A. et al. Desmoplastic Small Round Blue Cell Tumor: A Review of Treatment and Potential Therapeutic Genomic Alterations. Sarcoma 2017, 1278268, doi:10.1155/2017/1278268 (2017).

Shaffer, P. L., Jivan, A., Dollins, D. E., Claessens, F. \& Gewirth, D. T. Structural basis of androgen receptor binding to selective androgen response elements. Proc Natl Acad Sci U S A 101, 4758-4763, doi:10.1073/pnas.0401123101 (2004).

53 Pihlajamaa, P. et al. Tissue-specific pioneer factors associate with androgen receptor cistromes and transcription programs. EMBO J 33, 312-326, doi:10.1002/embj.201385895 (2014).

54 Sahu, B. et al. FoxA1 specifies unique androgen and glucocorticoid receptor binding events in prostate cancer cells. Cancer Res 73, 1570-1580, doi:10.1158/0008-5472.CAN-12-2350 (2013).

55 Sahu, B. et al. Dual role of FoxA1 in androgen receptor binding to chromatin, androgen signalling and prostate cancer. EMBO J 30, 3962-3976, doi:10.1038/emboj.2011.328 (2011).

56 Mirosevich, J. et al. Expression and role of Foxa proteins in prostate cancer. Prostate 66, 1013-1028, doi:10.1002/pros.20299 (2006).

57 Hingorani, P. et al. Transcriptome analysis of desmoplastic small round cell tumors identifies actionable therapeutic targets: a report from the Children's Oncology Group. Sci Rep 10, 12318, doi:10.1038/s41598020-69015-w (2020).

58 Edlind, M. P. \& Hsieh, A. C. PI3K-AKT-mTOR signaling in prostate cancer progression and androgen deprivation therapy resistance. Asian J Androl 16, 378-386, doi:10.4103/1008-682X.122876 (2014).

59 Qi, W. et al. Reciprocal feedback inhibition of the androgen receptor and PI3K as a novel therapy for castrate-sensitive and -resistant prostate cancer. Oncotarget 6, 41976-41987, doi:10.18632/oncotarget.5659 (2015). 
60 Michmerhuizen, A. R., Spratt, D. E., Pierce, L. J. \& Speers, C. W. ARe we there yet? Understanding androgen receptor signaling in breast cancer. NPJ Breast Cancer 6, 47, doi:10.1038/s41523-020-001909 (2020).

61 Fong, E. L. S. et al. Modeling Ewing sarcoma tumors in vitro with 3D scaffolds. Proceedings of the National Academy of Sciences of the United States of America 110, 6500-6505 (2013).

62 Lamhamedi-Cherradi, S. E. et al. IGF-1R and mTOR Blockade: Novel Resistance Mechanisms and Synergistic Drug Combinations for Ewing Sarcoma. Journal of the National Cancer Institute 108, doi:10.1093/jnci/djw182 (2016).

63 Puerto-Camacho, P. et al. Preclinical Efficacy of Endoglin-Targeting Antibody-Drug Conjugates for the Treatment of Ewing Sarcoma. Clin Cancer Res 25, 2228-2240, doi:10.1158/1078-0432.CCR-18-0936 (2019).

64 Dobin, A. et al. STAR: ultrafast universal RNA-seq aligner. Bioinformatics 29, 15-21, doi:10.1093/bioinformatics/bts635 (2013).

65 Anders, S., Pyl, P. T. \& Huber, W. HTSeq--a Python framework to work with high-throughput sequencing data. Bioinformatics 31, 166-169, doi:10.1093/bioinformatics/btu638 (2015).

66 Robinson, M. D., McCarthy, D. J. \& Smyth, G. K. edgeR: a Bioconductor package for differential expression analysis of digital gene expression data. Bioinformatics 26, 139-140, doi:10.1093/bioinformatics/btp616 (2010).

67 Ritchie, M. E. et al. limma powers differential expression analyses for RNA-sequencing and microarray studies. Nucleic Acids Res 43, e47, doi:10.1093/nar/gkv007 (2015).

68 Mootha, V. K. et al. PGC-1alpha-responsive genes involved in oxidative phosphorylation are coordinately downregulated in human diabetes. Nat Genet 34, 267-273, doi:10.1038/ng1180 (2003).

69 Wang, K. et al. MapSplice: accurate mapping of RNA-seq reads for splice junction discovery. Nucleic Acids Res 38, e178, doi:10.1093/nar/gkq622 (2010).

70 Terranova, C. et al. An Integrated Platform for Genome-wide Mapping of Chromatin States Using Highthroughput ChIP-sequencing in Tumor Tissues. Journal of visualized experiments : JoVE, doi:10.3791/56972 (2018).

71 Tang, M. pyflow-ChIPseq: a snakemake based ChIP-seq pipeline. Zenodo (2017).

72 Koster, J. \& Rahmann, S. Snakemake--a scalable bioinformatics workflow engine. Bioinformatics 28, 2520-2522, doi:10.1093/bioinformatics/bts480 (2012).

73 Langmead, B., Trapnell, C., Pop, M. \& Salzberg, S. L. Ultrafast and memory-efficient alignment of short DNA sequences to the human genome. Genome biology 10, R25, doi:10.1186/gb-2009-10-3-r25 (2009).

74 Ramirez, F. et al. deepTools2: a next generation web server for deep-sequencing data analysis. Nucleic acids research 44, W160-165, doi:10.1093/nar/gkw257 (2016).

75 Robinson, J. T. et al. Integrative genomics viewer. Nature biotechnology 29, 24-26, doi:10.1038/nbt.1754 (2011).

76 Zhang, Y. et al. Model-based analysis of ChIP-Seq (MACS). Genome biology 9, R137, doi:10.1186/gb2008-9-9-r137 (2008).

77 Yu, G., Wang, L. G. \& He, Q. Y. ChIPseeker: an R/Bioconductor package for ChIP peak annotation, comparison and visualization. Bioinformatics 31, 2382-2383, doi:10.1093/bioinformatics/btv145 (2015).

78 Loven, J. et al. Selective inhibition of tumor oncogenes by disruption of super-enhancers. Cell 153, 320334, doi:10.1016/j.cell.2013.03.036 (2013).

79 Khan, A. \& Mathelier, A. Intervene: a tool for intersection and visualization of multiple gene or genomic region sets. BMC Bioinformatics 18, 287, doi:10.1186/s12859-017-1708-7 (2017).

80 Yu, G., Wang, L. G., Han, Y. \& He, Q. Y. clusterProfiler: an R package for comparing biological themes among gene clusters. OMICS 16, 284-287, doi:10.1089/omi.2011.0118 (2012).

$81 \mathrm{Yu}, \mathrm{G}$. enrichplot: Visualization of Functional Enrichment Result. $R$ package version 1.6.1, https://github.com/GuangchuangYu/enrichplot. (2019). 
bioRxiv preprint doi: https://doi.org/10.1101/2022.01.20.477146; this version posted January 22, 2022. The copyright holder for this preprint (which was not certified by peer review) is the author/funder, who has granted bioRxiv a license to display the preprint in perpetuity. It is made available under aCC-BY-NC-ND 4.0 International license.

82 Heinz, S. et al. Simple combinations of lineage-determining transcription factors prime cis-regulatory elements required for macrophage and B cell identities. Mol Cell 38, 576-589, doi:10.1016/j.molcel.2010.05.004 (2010).

83 Subudhi, S. K. et al. Neoantigen responses, immune correlates, and favorable outcomes after ipilimumab treatment of patients with prostate cancer. Sci Transl Med 12, doi:10.1126/scitranslmed.aaz3577 (2020). 
Figure 1

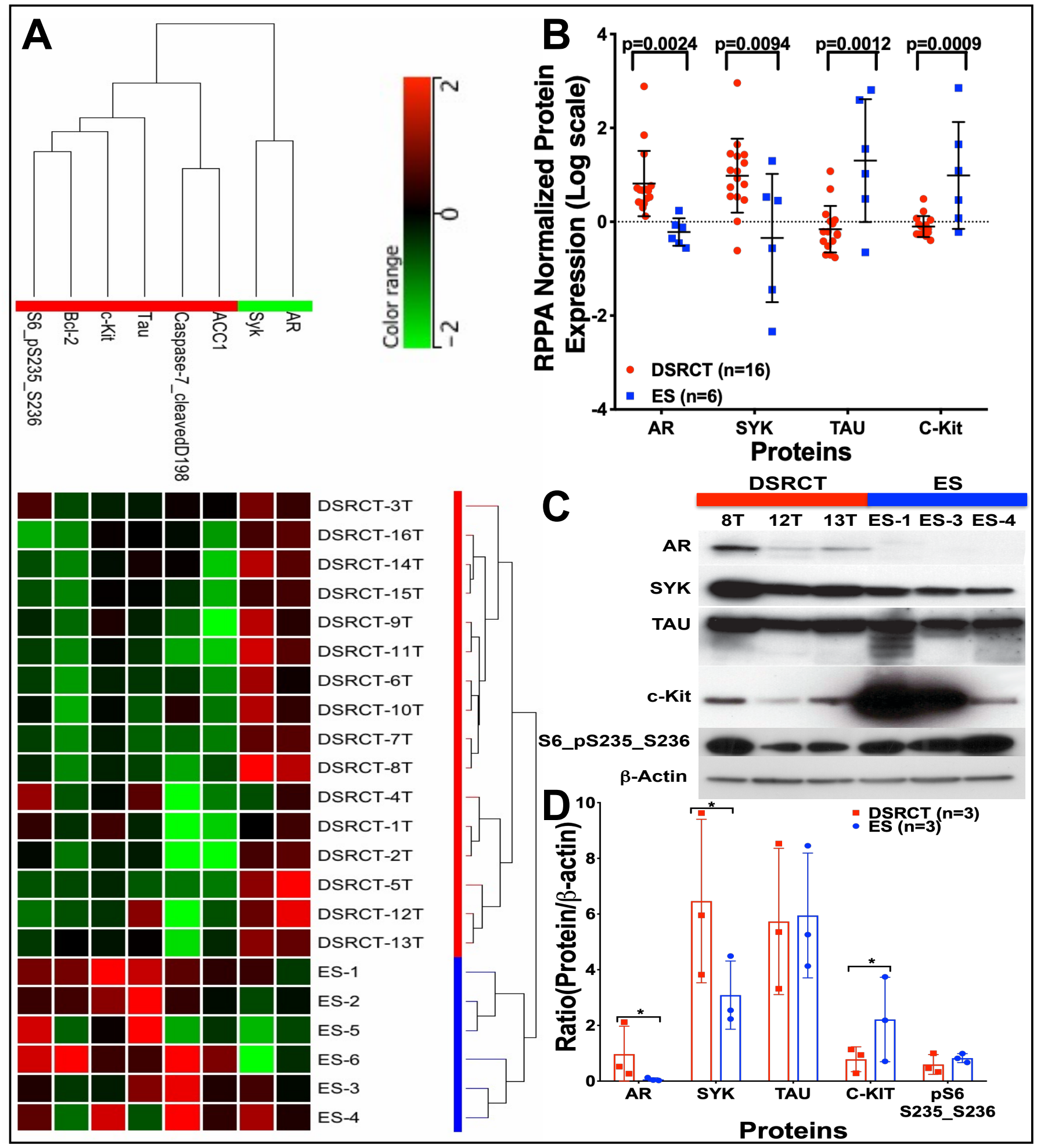


bioRxiv preprint doi: https://doi.org/10.1101/2022.01.20.477146; this version posted January 22, 2022. The copyright holder for this preprint (which was not certified by peer review) is the author/funder, who has granted bioRxiv a license to display the preprint in perpetuity. It is made available under aCC-BY-NC-ND 4.0 International license.

\section{Figure 2}

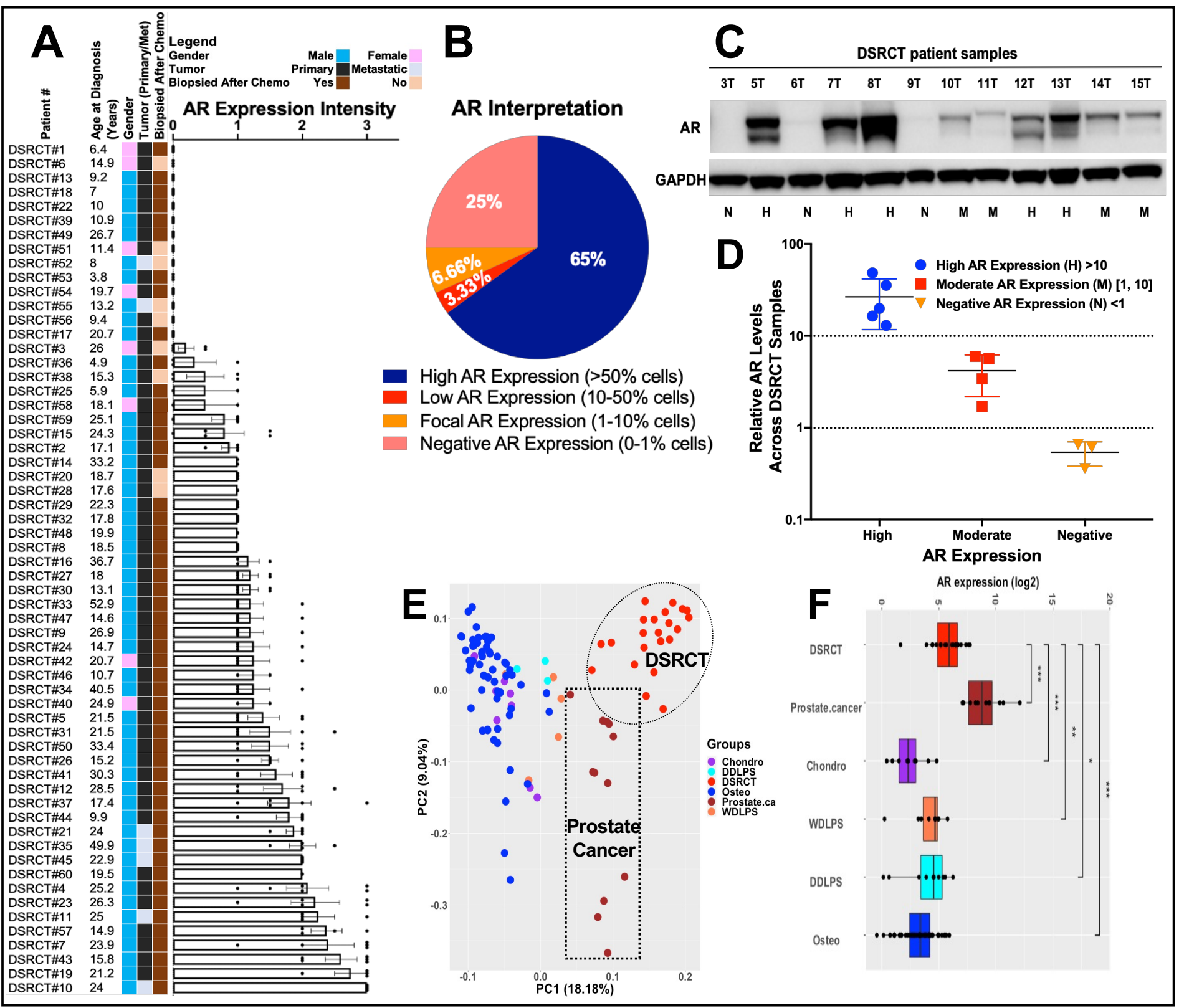




\section{Figure 3}

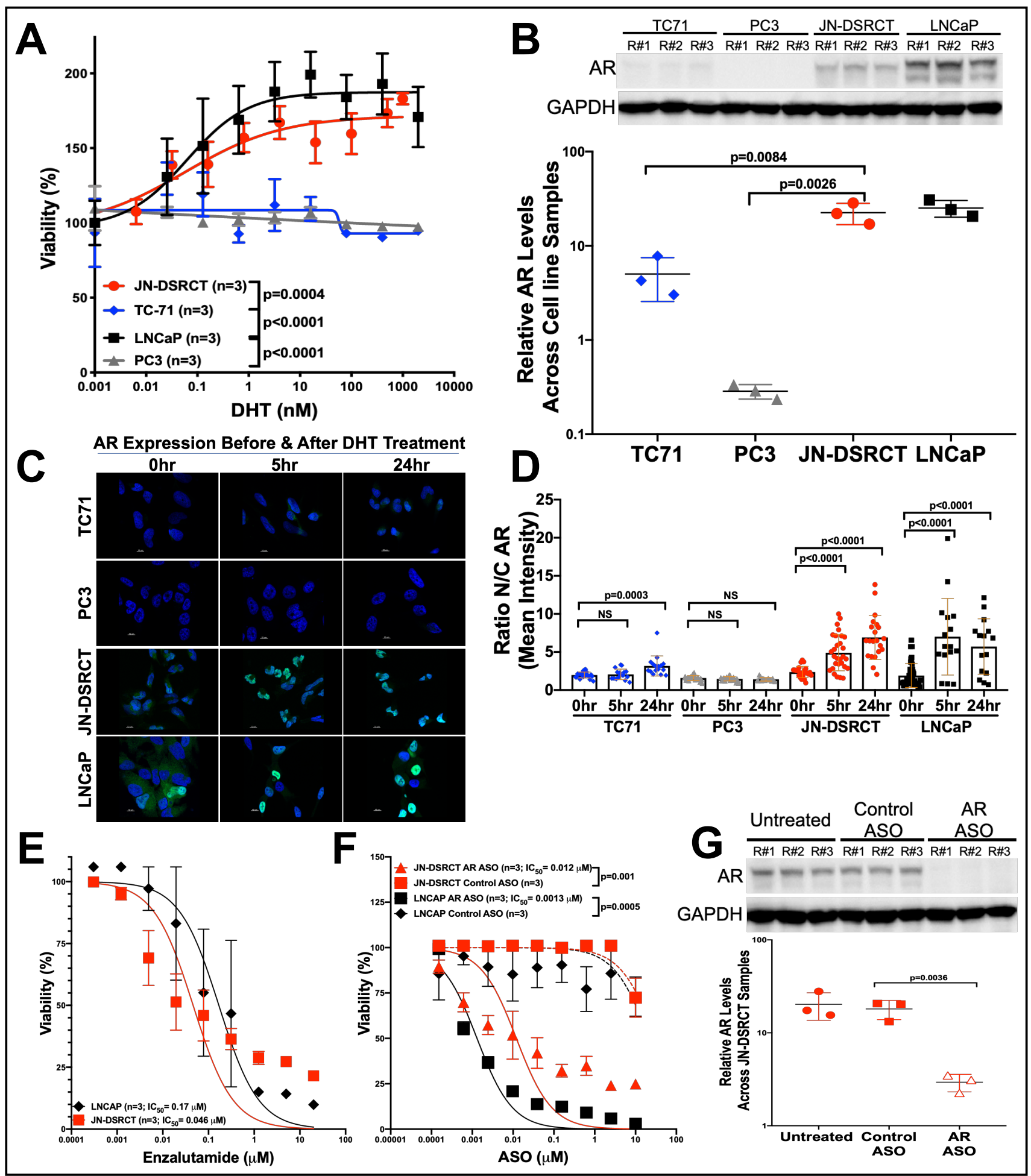


bioRxiv preprint doi: https://doi.org/10.1101/2022.01.20.477146; this version posted January 22, 2022. The copyright holder for this preprint (which was not certified by peer review) is the author/funder, who has granted bioRxiv a license to display the preprint in perpetuity. It is made available under aCC-BY-NC-ND 4.0 International license.

Figure 4

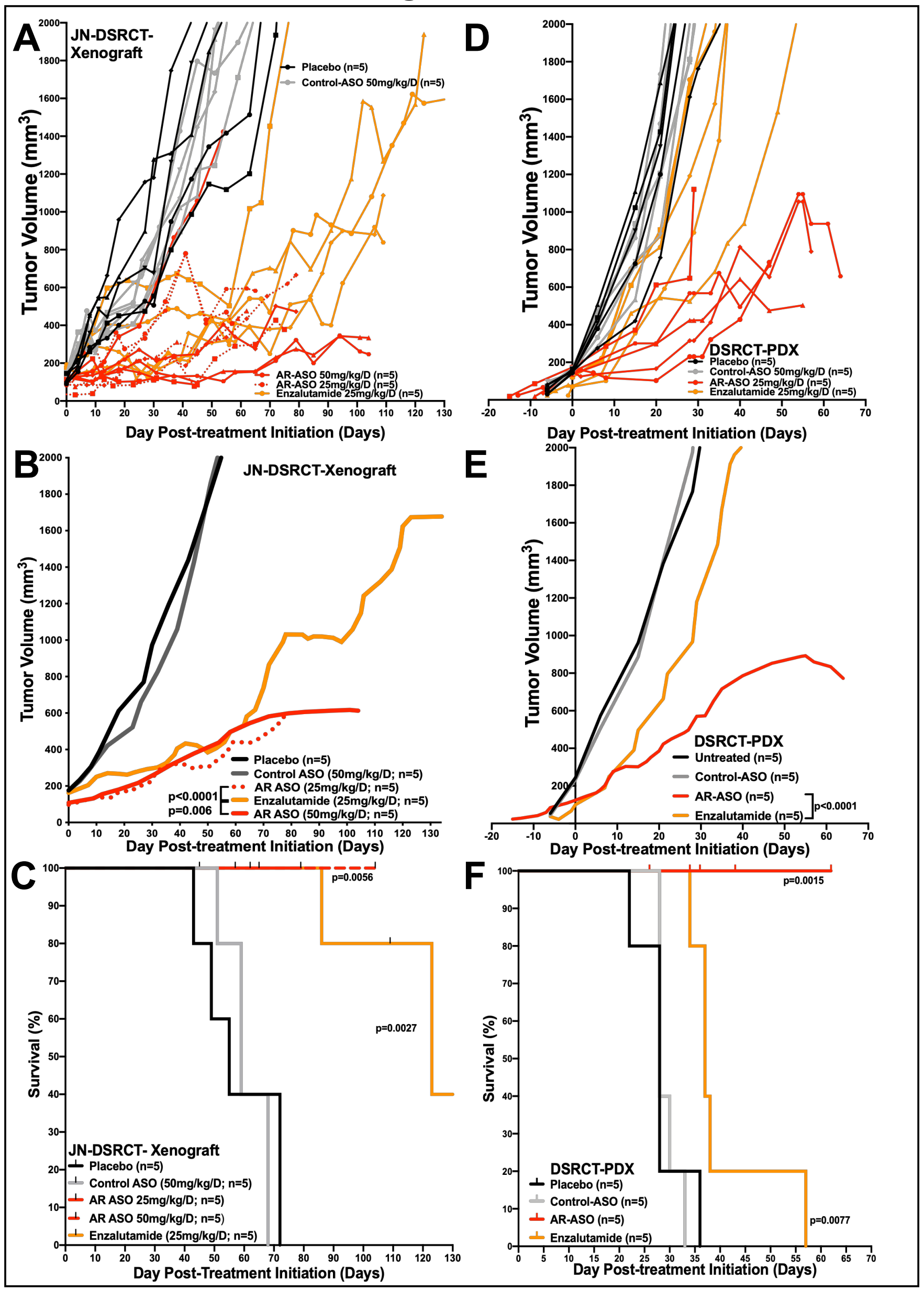


bioRxiv preprint doi: https://doi.org/10.1101/2022.01.20.477146; this version posted January 22, 2022. The copyright holder for this preprint (which was not certified by peer review) is the author/funder, who has granted bioRxiv a license to display the preprint in perpetuity. It is made available under aCC-BY-NC-ND 4.0 International license.

\section{Figure 5}

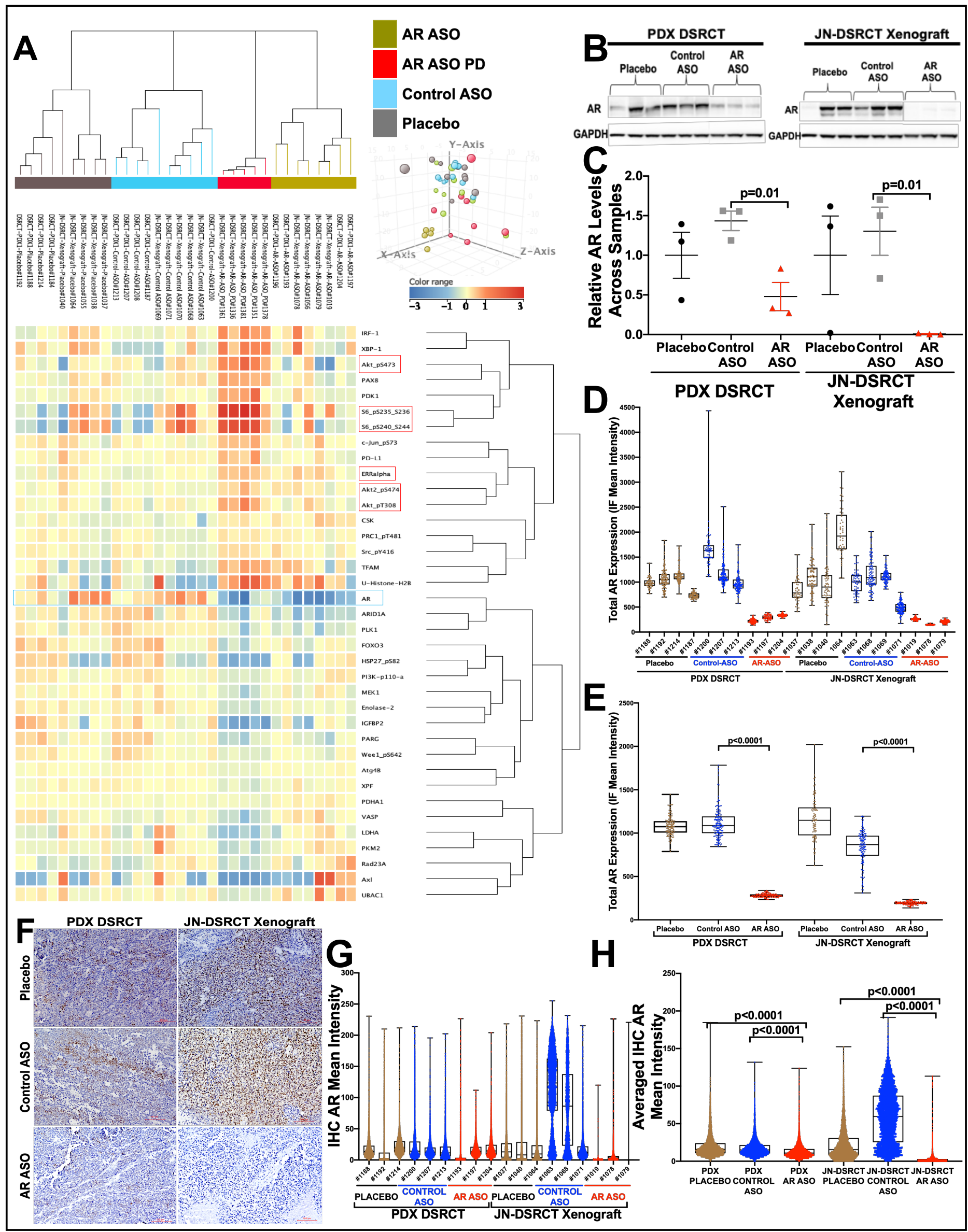




\section{Figure 6}

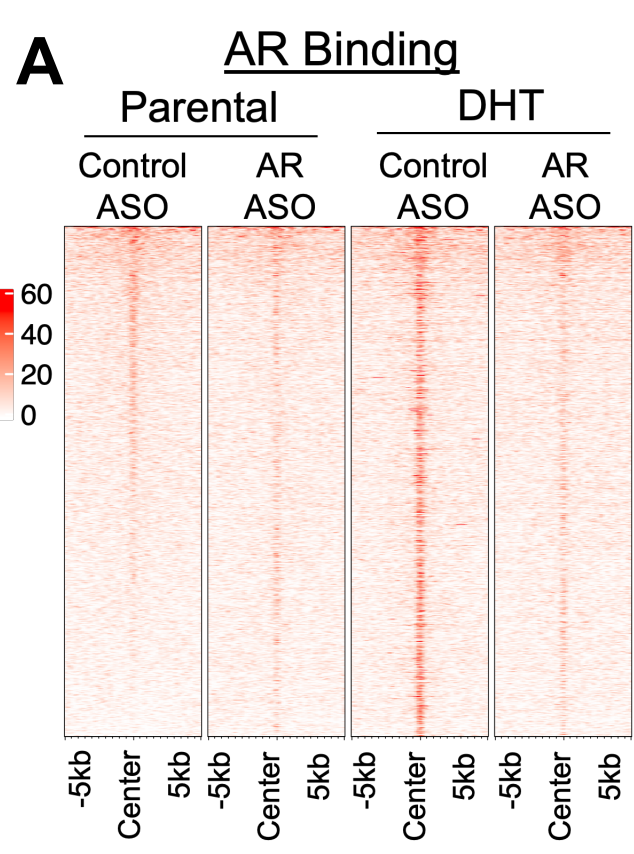

C

\begin{tabular}{|c|c|c|c|}
\hline \multicolumn{4}{|c|}{ AR specific binding Motifs } \\
\hline \multirow[t]{2}{*}{ TF Class } & \multicolumn{2}{|l|}{ TF } & \multirow{2}{*}{$\begin{array}{c}\text { P-value } \\
\text { 1e-174 }\end{array}$} \\
\hline & IAAGTIAAACA & FOXA1 & \\
\hline \multirow[t]{2}{*}{ Forkhead } & TATTTACITA & FOXM1 & 1e-169 \\
\hline & 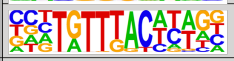 & FOXA3 & 1e-157 \\
\hline \multirow{3}{*}{$\begin{array}{l}\text { Nuclear } \\
\text { receptor }\end{array}$} & 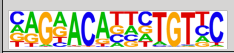 & GRE & $1 e-151$ \\
\hline & ACACAETCTICTCE & ARE & 1e-143 \\
\hline & ACACACATAATATTC & PGR & 1e-119 \\
\hline \multirow{3}{*}{$\begin{array}{l}\text { Zinc } \\
\text { finger }\end{array}$} & СCTCCCACACAT & WT1 & $1 e-55$ \\
\hline & TEGGTGGG'CG & Egr1 & 1e-29 \\
\hline & 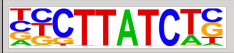 & Gata2 & 1e-24 \\
\hline
\end{tabular}

$\mathbf{E}$

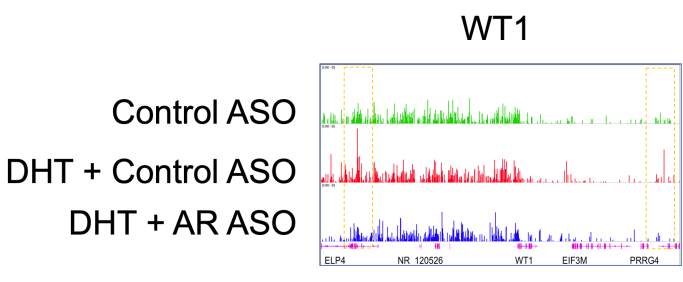

GATA6

Control ASO

DHT + Control ASO

$\mathrm{DHT}+\mathrm{AR}$ ASO

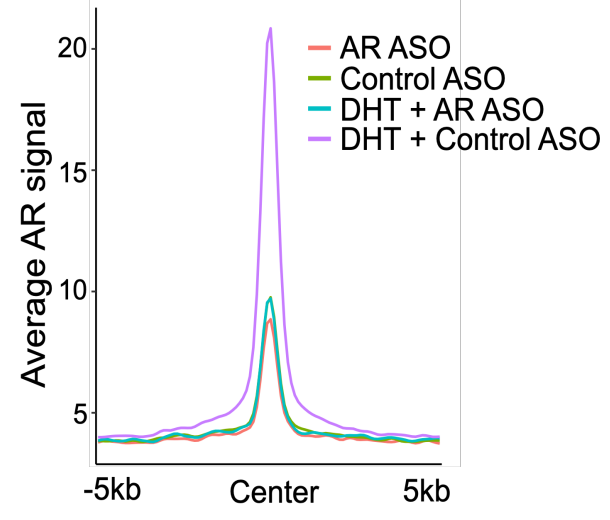

D

AR specific Upregulated genes in linked genes

Control ASO

DHT +

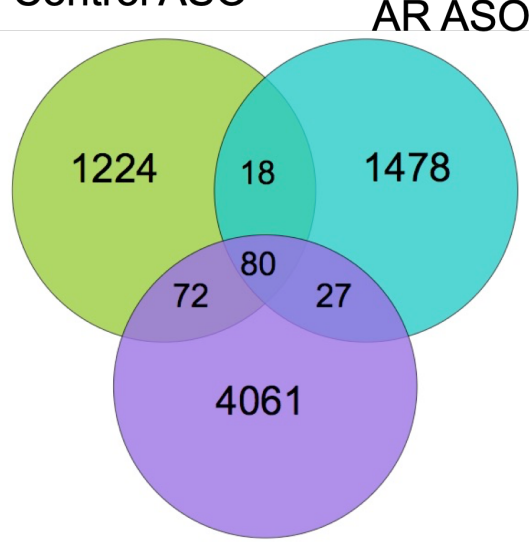

DHT +

Control ASO 


\section{Figure 7}

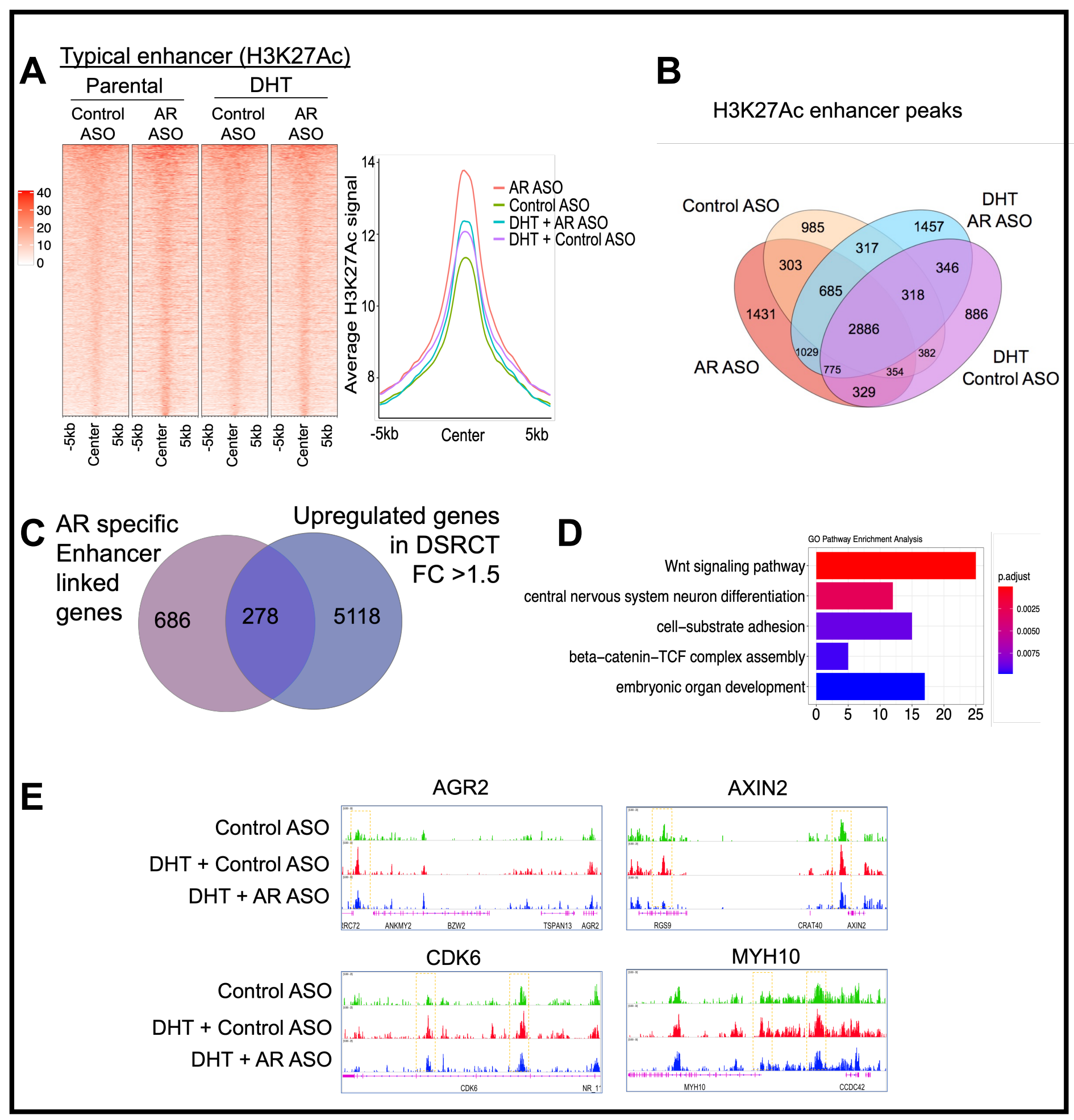

